Check for updates

Cite this: RSC Adv., 2021, 11, 8107

Received 16th November 2020 Accepted 11th February 2021

DOI: $10.1039 / \mathrm{dOra09712h}$

rsc.li/rsc-advances

\title{
An ingenious method for the determination of the relative and absolute configurations of compounds containing aryl-glycerol fragments by ${ }^{1} \mathrm{H}$ NMR spectroscopy $\dagger$
}

\author{
Xu Zhang, Kai-Zhou Lu, Hai-Wei Yan, Zi-Ming Feng, Ya-Nan Yang, (iD \\ Jian-Shuang Jiang and Pei-Cheng Zhang (D)
}

\begin{abstract}
A concise method was established to determine the relative and absolute configurations of aryl-glycerols that depend on the chemical shift differences $(\Delta \delta)$ of the diastereotopic methylene protons $(\mathrm{H}-3)$ by ${ }^{1} \mathrm{H}$ NMR spectroscopy. When using $\mathrm{DMSO}-d_{6}$ as the preferred solvent, the threo configuration corresponded to a larger $\Delta \delta_{\mathrm{H} 3 \mathrm{a}-\mathrm{H} 3 \mathrm{~b}}$ value $(>0.15 \mathrm{ppm})$, whereas the erythro configuration $(<0.07 \mathrm{ppm})$ corresponded to a smaller value. Furthermore, the absolute configurations were determined with the aid of a simple acylation reaction through camphanoyl chloride. In the threo enantiomers, the $\Delta \delta$ value of the $1 R, 2 R$ configuration was $<0.15 \mathrm{ppm}$, and that of the $1 S, 2 S$ configuration was $>0.20 \mathrm{ppm}$. In the erythro enantiomers, the $\Delta \delta$ value of $1 R, 2 S$ was $>0.09 \mathrm{ppm}$, and that of $1 S, 2 R$ was $<0.05 \mathrm{ppm}$. Remarkably, this empirical rule is invalid in $\mathrm{CDCl}_{3}$. In addition, this method was also verified by a quantum ${ }^{1} \mathrm{H}$ NMR calculation.
\end{abstract}

\section{Introduction}

Chiral alcohols, such as chiral secondary alcohols, prim,secdiols, sec,sec-diols and 1,2,3-prim,sec,sec-triols, are ubiquitous in natural products and synthetic products. The assignment of their relative and absolute configurations is always a challenging task. Because of the difficulty of growing crystals of these compounds and the lack of distinct Cotton effects in their electronic circular dichroism (ECD) spectra, NMR spectroscopy is suitable for this purpose and is readily available compared with other methods. For instance, the absolute configurations of chiral secondary alcohols were determined using Mosher's method by ${ }^{1} \mathrm{H}$ NMR spectroscopy; ${ }^{1}$ the relative configurations of some sec,sec-diols were assigned using a JBCA ( $\mathrm{J}$-based configuration analysis) method ${ }^{2}$ or by comparison of their ${ }^{13} \mathrm{C}-\Delta \delta$ behaviors in a chiral bidentate NMR solvent. ${ }^{3}$ We have also developed two approaches to (1) discriminate the threo and erythro configurations of vicinal diol in polyacetylene glycosides by the ${ }^{3} J_{\mathrm{HH}}$ value using acetic acid- $d_{4} / \mathrm{D}_{2} \mathrm{O}$ as the solvent; ${ }^{4}$ and (2) determine the absolute configurations of 2-hydroxy phenylethanoid glycosides (prim,sec-diol derivatives) by the chemical shift differences $(\Delta \delta)$ of the diastereotopic methylene protons. ${ }^{5}$

State Key Laboratory of Bioactive Substance and Function of Natural Medicines, Institute of Materia Medica, Chinese Academy of Medical Sciences, Peking Union Medical College, Beijing 100050, People's Republic of China. E-mail: pczhang@imm. ac.cn

$\dagger$ Electronic supplementary information (ESI) available: 1D NMR, 2D NMR, and HRMS spectra. See DOI: 10.1039/d0ra09712h
Aryl-glycerols (AGs), a kind of 1,2,3-prim,sec,sec-triols, are frequent in natural products such as phenylpropanoids and lignans ${ }^{6-15}$ and are important intermediates in many chemical syntheses. ${ }^{16-21}$ Due to the conformational flexibility of the triol chain in AGs, determining the relative and absolute configurations is significantly challenging. Recently, Mosher's method was developed to assign the absolute configuration of AGs by comparing the ${ }^{1} \mathrm{H}$ NMR data of the tris- $(R)$ - and the tris- $(S)$-MPA ester derivatives with those of the AGs. ${ }^{22,23}$ However, the inability to prepare tris-MPA ester derivatives for trace compounds and the complex rules governing the comparison of the $\Delta \delta^{R S}(\mathrm{H}-1)$ and $\left|\Delta \delta^{R S}{ }_{\mathrm{H}(2)}-\Delta \delta^{R S}{ }_{\mathrm{H}(1)}\right|$ of AGs, to some extent, have limited the application of this method. For some naturally occurring AGs, the regular $\Delta \delta_{\mathrm{C} 1-\mathrm{C} 2}$ parameters have been used to differentiate only the threo and erythro relative<smiles>OCC(O)C(O)O</smiles><smiles>OC(O)C(O)c1ccccc1</smiles><smiles>[R3]C(CO)C(O)c1ccccc1</smiles><smiles>OC(O)C(O)c1ccccc1</smiles>

Fig. 1 Structures of compounds M1-M4 
<smiles>COc1cc([C@@H](O)[C@H](O)CO)cc(OC)c1O</smiles><smiles>COc1cc([C@@H](O)[C@H](O)CO)cc(OC)c1O</smiles><smiles>OC[C@@H](O)[C@H](O)c1ccc(Br)cc1</smiles><smiles>OC[C@@H](O)[C@H](O)c1ccc(Br)cc1</smiles><smiles>OC[C@@H](O)[C@H](O)c1ccc(Br)cc1</smiles><smiles>OCC(O)[C@H](O)c1ccc(Br)cc1</smiles><smiles>O=[N+]([O-])c1ccc([C@@H](O)[C@H](O)CO)cc1</smiles><smiles>O=[N+]([O-])c1ccc([C@@H](O)[C@H](O)CO)cc1</smiles><smiles>COc1cc([C@@H](O)[C@H](O)CO)ccc1O</smiles><smiles>COc1cc([C@@H](O)[C@@H](O)CO)ccc1O</smiles><smiles>COc1cc([C@@H]2Oc3c(OC)cc([C@@H](O[C@@H](O)CO)[C@H](O)CO)cc3C2CO)cc(OC)c1O</smiles>

Fig. 2 Structures of compounds 1-11.

configurations. ${ }^{9,15}$ However, we found that this rule is not applicable to AGs without substituent groups on the benzene ring. Therefore, a new reliable method to determine the relative and absolute configurations of AGs is a worthwhile task.

\section{Results and discussion}

Our recent study found that the threo and erythro configurations of $\mathrm{H}-7$ and $\mathrm{H}-8$ in 8,4'-oxyneolignans can be discriminated by the values of $\Delta \delta_{\mathrm{H} 9 \mathrm{a}-\mathrm{H} 9 \mathrm{~b}}$ of methylene protons in ${ }^{1} \mathrm{H}$ NMR spectroscopy. ${ }^{24}$ When we determined the configurations of a pair of threo syringylglycerol enantiomers ( 1 and 2 ) obtained from the Arnebia euchroma, a careful comparison of the ${ }^{1} \mathrm{H}$ NMR spectroscopic data of $\mathbf{1}$ in methanol- $d_{4}$ with the corresponding data in erythro syringylglycerol in the literature showed that there were significant differences. ${ }^{9}$ The value of $\Delta \delta_{\mathrm{H} 3 \mathrm{a}-\mathrm{H} 3 \mathrm{~b}}$ of methylene protons in 1 is $0.13 \mathrm{ppm}$, while the value is only $0.07 \mathrm{ppm}$ in erythro syringylglycerol. Surprisingly, this result is similar to the rule in 8,4'-oxyneolignans. For AGs without large steric hindrance groups, can the $\Delta \delta_{\mathrm{H} 3 \mathrm{a}-\mathrm{H} 3 \mathrm{~b}}$ values of the methylene groups reflect their relative stereochemistry? To explore and

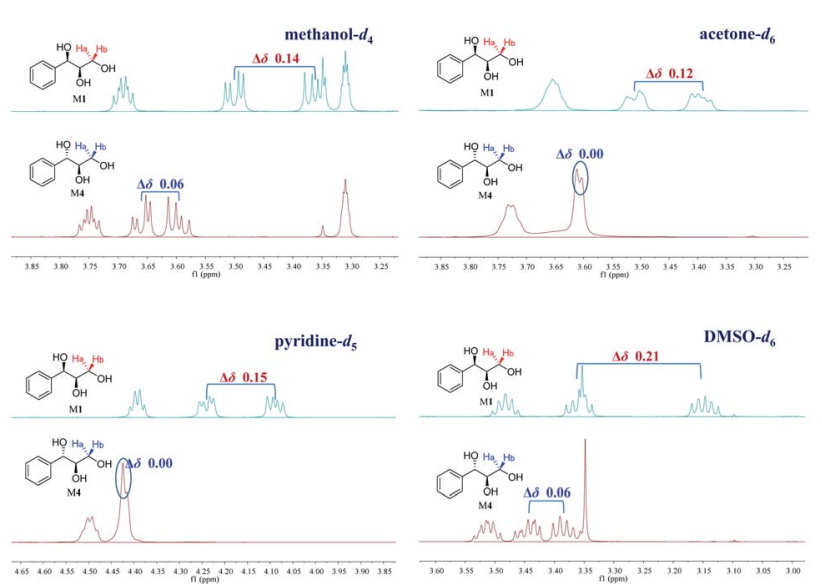

Fig. 3 Influence of different deuterated solvents on the $\Delta \delta_{\mathrm{H} 3 \mathrm{a}-\mathrm{H} 3 \mathrm{~b}}$ values of $\mathrm{M} 1$ and $\mathrm{M} 4\left({ }^{1} \mathrm{H}\right.$ NMR $\left.500 \mathrm{MHz}\right)$. summarize this phenomenon, two pairs of 1-phenylpropane1,2,3-triol enantiomers were prepared (M1-M4) through the hydrolysis of $(2 R, 3 R)$-3-phenyl-2-oxiranemethanol and $(2 S, 3 S)$-3phenyl-2-oxiranemethanol (Fig. 1). The ${ }^{1} \mathrm{H}$ NMR spectra of compounds M1 and M4 in methanol- $d_{4}$ were recorded. Obviously, the $\Delta \delta_{\mathrm{H} 3 \mathrm{a}-\mathrm{H} 3 \mathrm{~b}}(0.14 \mathrm{ppm})$ value of methylene protons in the threo configuration was remarkably larger than the corresponding value (0.06 ppm) in the erythro configuration (Fig. 3$)$. Considering that deuterated solvents have a major impact on the chemical shifts of methylene protons, the ${ }^{1} \mathrm{H}$ NMR spectra of compounds M1 and M4 were recorded in other deuterated solvents, acetone- $d_{6}$, pyridine- $d_{5}$, DMSO- $d_{6}$, and $\mathrm{CDCl}_{3}$, as shown in Fig. 3. Similarly, in the threo configurations, the $\Delta \delta_{\mathrm{H} 3 \mathrm{a}-\mathrm{H} 3 \mathrm{~b}}$ values of methylene protons were 0.12 in acetone- $d_{6}$, 0.15 in pyridine- $d_{5}$, and 0.21 in DMSO- $d_{6}$. In the erythro configurations, the $\Delta \delta_{\mathrm{H} 3 \mathrm{a}-\mathrm{H} 3 \mathrm{~b}}$ values of methylene protons were 0.00 in acetone- $d_{6}, 0.00$ in pyridine- $d_{5}$, and 0.06 in DMSO- $d_{6}$. Remarkably, there was no obvious difference in $\mathrm{CDCl}_{3}$ (threo:

Table $1 \Delta \delta_{\mathrm{H} 3 a-\mathrm{H} 3 \mathrm{~b}}$ values (ppm) of the AGs 1-11 in different solvents $\left({ }^{1} \mathrm{H}\right.$ NMR $\left.500 \mathrm{MHz}\right)$

\begin{tabular}{llllll}
\hline No. & $\delta_{\mathrm{H} 3 \mathrm{a}}$ & $\delta_{\mathrm{H} 3 \mathrm{~b}}$ & $\Delta \delta_{\mathrm{H} 3 \mathrm{a}-\mathrm{H} 3 \mathrm{~b}}$ & Solvent & Relative conf. \\
\hline $\mathbf{1} \& \mathbf{2}$ & 3.50 & 3.37 & 0.13 & Methanol- $d_{4}$ & Threo \\
& 3.49 & 3.40 & 0.09 & Acetone- $d_{6}$ & \\
$\mathbf{3} \& \mathbf{4}$ & 3.55 & 3.39 & 0.16 & Methanol- $d_{4}$ & Threo \\
& 3.56 & 3.42 & 0.14 & Acetone- $d_{6}$ & \\
& 4.27 & 4.09 & 0.18 & Pyridine- $d_{5}$ & \\
& 3.39 & 3.15 & 0.24 & DMSO- $d_{6}$ & \\
$\mathbf{5} \& \mathbf{6}$ & 3.64 & 3.59 & 0.05 & Methanol- $d_{4}$ & Erythro \\
& 3.61 & 3.61 & 0.00 & Acetone- $d_{6}$ & \\
& 4.39 & 4.39 & 0.00 & Pyridine- $d_{5}$ & \\
$\mathbf{7} \& \mathbf{8}$ & 3.42 & 3.37 & 0.05 & DMSO- $d_{6}$ & \\
& 3.68 & 3.65 & 0.03 & Methanol- $d_{4}$ & Erythro \\
& 3.67 & 3.63 & 0.04 & Acetone- $d_{6}$ & \\
$\mathbf{9} \& \mathbf{1 0}$ & 3.42 & 3.42 & 0.00 & DMSO- $d_{6}$ & \\
& 3.47 & 3.34 & 0.13 & Methanol- $d_{4}$ & Threo \\
$\mathbf{1 1}$ & 3.31 & 3.14 & 0.17 & DMSO- $d_{6}$ & \\
& 3.52 & 3.38 & 0.14 & Methanol- $d_{4}$ & Threo \\
& 3.35 & 3.18 & 0.17 & DMSO- $d_{6}$ &
\end{tabular}




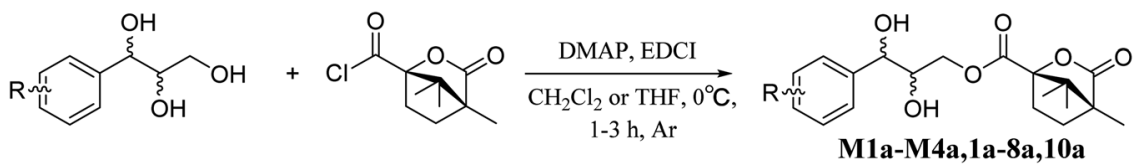

Scheme 1 The acylation reaction.

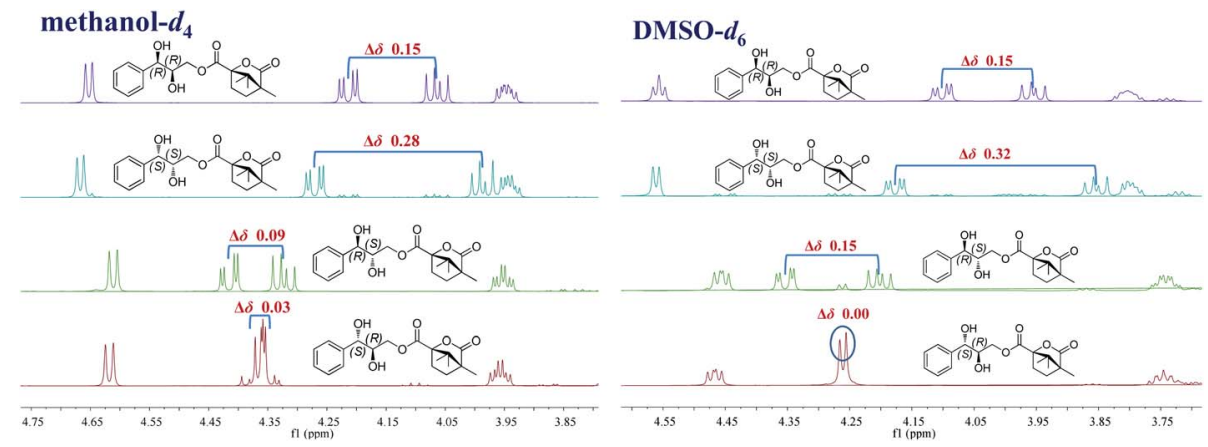

Fig. 4 Influence of different deuterated solvents on the $\Delta \delta_{\mathrm{H} 3 \mathrm{a}-\mathrm{H} 3 \mathrm{~b}}$ values of $\mathrm{M} 1 \mathrm{a}-\mathrm{M} 4 \mathrm{a}\left({ }^{1} \mathrm{H} \mathrm{NMR} 500 \mathrm{MHz}\right)$.

$0.09 \mathrm{ppm}$; erythro: $0.07 \mathrm{ppm})$. In addition, the chemical shift values of methylene protons in the erythro configuration were obviously larger than those in the threo configuration.

To further confirm the accuracy and reliability of the method and investigate the influence of different substitution groups on the benzene ring, 1-(4-bromophenyl)propane-1,2,3-triol (3-6) and 1-(4-nitrophenyl)propane-1,2,3-triol (7 and 8) were synthesized through the Sharpless asymmetric epoxidation and hydrolysis reaction (Fig. 2). ${ }^{25}$ As expected, larger $\Delta \delta_{\mathrm{H} 3 \mathrm{a}-\mathrm{H} 3 \mathrm{~b}}$ values of methylene protons appeared in the threo configurations (3-4), and smaller $\Delta \delta_{\mathrm{H} 3 \mathrm{a}-\mathrm{H} 3 \mathrm{~b}}$ values of methylene protons appeared in the erythro configurations (5-8). Additionally, three natural products, threo-1-C-guaiacylglycerol ( 9 and $\mathbf{1 0})$, obtained from bamboo juice, and compound 11, obtained from Cortex Lycii, were also in accordance with the general trends in methanol- $d_{4}$ and DMSO- $d_{6}$ (Fig. 2). Thus, the relative configuration of AGs can be determined from the $\Delta \delta_{\mathrm{H} 3 \mathrm{a}-\mathrm{H} 3 \mathrm{~b}}$ values of methylene protons of the ${ }^{1} \mathrm{H}$ NMR spectra in methanol- $d_{4}$, acetone- $d_{6}$, pyridine- $d_{5}$, and DMSO- $d_{6}$; DMSO- $d_{6}$ is the preferred solvent (Table 1).
Then, a more challenging task is how to determine the absolute configurations of a pair of enantiomers. Considering the conformational flexibility of the triol chain in AGs, the introduction of a chiral auxiliary agent bearing a larger steric hindrance group may be an efficient approach. Camphanoyl chloride, a acylating agent containing two special stereogenic centers, is frequently utilized to determine enantiomeric purity in organic synthesis and its derivatives was also used to assign absolute configurations of chiral alcohols by NMR. ${ }^{26,27}$ It made molecules more rigid due to its steric hindrance in conformational studies. These features inspired us to thoroughly investigate the relationship between the chemical shift differences of methylene protons and the absolute configurations of monocamphanoyl AGs.

Accordingly, acylation reactions (Scheme 1) were carried out using (-)-1S,4R-camphanoyl chloride and two pairs of 1-phenyl glycerol enantiomers M1-M4 as raw materials, and four products (M1a-M4a) were successfully obtained. Their ${ }^{1} \mathrm{H}$ NMR spectra were measured in several deuterated solvents, including methanol- $d_{4}$, DMSO- $d_{6}$, acetone- $d_{6}$, pyridine- $d_{5}$, and $\mathrm{CDCl}_{3}$. To our delight, in methanol- $d_{4}$ and DMSO- $d_{6}$, the $\Delta \delta_{\mathrm{H} 3 \mathrm{a}-\mathrm{H} 3 \mathrm{~b}}$ values
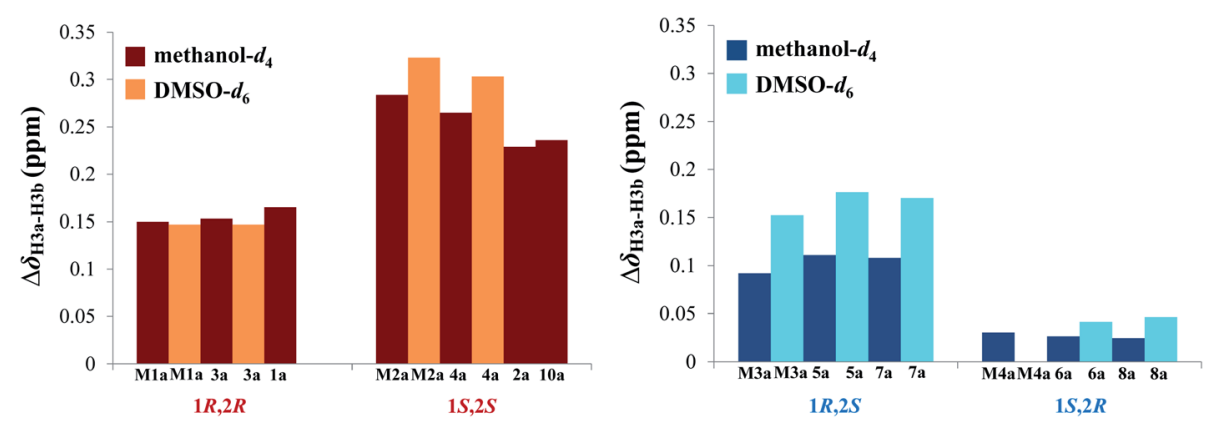

Fig. $5 \Delta \delta_{\mathrm{H} 3 \mathrm{a}-\mathrm{H} 3 \mathrm{~b}}$ values $(\mathrm{ppm})$ of the acylation products. 
of methylene protons showed obvious differences in the threo and erythro configurations. In the threo enantiomers, the $\Delta \delta_{\mathrm{H} 3 \mathrm{a}-}$ H3b values of methylene protons in M1a $(1 R, 2 R)$ were 0.15 and 0.15 , respectively, while the $\Delta \delta_{\mathrm{H} 3 \mathrm{a}-\mathrm{H} 3 \mathrm{~b}}$ values in M2a $(1 S, 2 S)$ were 0.28 and 0.32 , respectively (Fig. 4). In the erythro system, the $\Delta \delta_{\mathrm{H} 3 \mathrm{a}-\mathrm{H} 3 \mathrm{~b}}$ values of methylene protons in M3a $(1 R, 2 S)$ were 0.09 and 0.15 , respectively, while the $\Delta \delta_{\mathrm{H} 3 \mathrm{a}-\mathrm{H} 3 \mathrm{~b}}$ values in M4a $(1 S, 2 R)$ were 0.03 and 0.00 , respectively. The chemical shift differences were high enough to discriminate the absolute configuration, especially when using DMSO- $d_{6}$ as the solvent. However, in the other three deuterated solvents, acetone- $d_{6}$, $\mathrm{CDCl}_{3}$, and pyridine- $d_{5}$, the $\Delta \delta_{\mathrm{H} 3 \mathrm{a}-\mathrm{H} 3 \mathrm{~b}}$ values were irregular. These results suggested that the anisotropic effect of camphanoyl could be efficiently and selectively space oriented toward the methylene protons of mono-camphanoyl AGs in methanol$d_{4}$ and DMSO- $d_{6}$. To further verify the reliability of the method, derivatives 1a-8a, and 10a were also successfully synthesized. As expected, the rule still applies in methanol- $d_{4}$ and DMSO- $d_{6}$ (Fig. 5). Furthermore, the theoretical ${ }^{1} \mathrm{H}$ NMR calculation of M1a-M4a was carried out by GIAO NMR calculation method in DMSO (the detailed procedure see $\mathrm{ESI} \dagger$ ). ${ }^{28}$ The calculated results were better match with the experimental data $\left(R^{2} \geq\right.$ 0.995) (Fig. 6). The calculated $\Delta \delta_{\mathrm{H} 3 \mathrm{a}-\mathrm{H} 3 \mathrm{~b}}$ values were M1a: 0.08 , M2a: 0.37, M3a: 0.72, and M4a: 0.06, respectively. This result verified our method was also reliable by quantum chemical calculation. These results confirmed that camphanoyl chloride is a suitable pure chiral derivatizing agent to assign the absolute configuration of AGs.

\section{Experimental section}

\section{General experimental procedures}

The optical rotations were recorded with a RUDOLPH automatic V polarimeter (RUDOLPH, Hackettstown, NJ, USA). The NMR spectra were recorded with a Bruker $500 \mathrm{MHz}$ (Bruker-Biospin, Billerica, MA, USA). HRESIMS reports were obtained from an Agilent 6520 HPLC-Q-TOF (Agilent Technologies, Waldbronn, Germany) and Q Exactive Focus LCMSMS (Thermo Scientific, MA, USA). Preparative HPLC separations were performed using a Shimadzu LC-10AT with an ODS-A column $(250 \mathrm{~mm} \times 10$ mm, $5 \mu \mathrm{m}$; YMC Corp., Kyoto, Japan). An Agilent 1200 series system with an Apollo C 18 column $(250 \mathrm{~mm} \times 4.6 \mathrm{~mm}, 5 \mu \mathrm{m}$; Alltech Corp., KY, USA) was used for HPLC-DAD analysis. All reactions were magnetically stirred with a DF-101S magnetic stirrer (Hengfengchangwei Technology Co. Ltd, Beijing, China). Commercially available reagents were used without further purification unless otherwise stated. All reactions were monitored by TLC with silica gel pre-coated GF254 plates and HPLCDAD.

\section{Structure characterization and synthesis procedures}

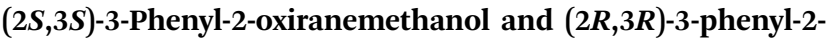
oxiranemethanol. The asymmetric epoxidation was carried out according to the Sharpless asymmetric Epoxidation. Colorless oil; the specific rotations were $[\alpha]_{\mathrm{D}}^{20}-47$ (c 0.1 methanol) and $[\alpha]_{\mathrm{D}}^{20}+70\left(c 0.1\right.$ methanol), respectively; ${ }^{1} \mathrm{H}$-NMR (500 $\mathrm{MHz}$, methanol- $\left.d_{4}\right): \delta 7.27-7.35$ (5H, one mono-substituted benzene ring system), $3.87(1 \mathrm{H}, \mathrm{dd}, J=12.5,3.0 \mathrm{~Hz}), 3.83$ $(1 \mathrm{H}, \mathrm{d}, J=2.0 \mathrm{~Hz}), 3.67(1 \mathrm{H}, \mathrm{dd}, J=12.5,5.5 \mathrm{~Hz}), 3.15(1 \mathrm{H}$, $\mathrm{m}) ;{ }^{13} \mathrm{C}-\mathrm{NMR}\left(125 \mathrm{MHz}, \mathrm{CDCl}_{3}\right): \delta 136.8,128.7,128.7,128.5$, 125.9, 125.9, 62.5, 61.3, 55.7; HRESIMS $m / z 151.0754[\mathrm{M}+$ $\mathrm{H}]^{+}$(calcd for $\mathrm{C}_{9} \mathrm{H}_{11} \mathrm{O}_{2}$ 151.0753).

A solution of $(2 R, 3 R)$-3-phenyl-2-oxiranemethanol $(100 \mathrm{mg}$, $0.6 \mathrm{mmol})$ and acetic acid $(0.5 \mathrm{ml})$ in acetonitrile was stirred by rotary evaporator at $55{ }^{\circ} \mathrm{C}$ until the solution was dry. Through the analysis of HPLC, the $(2 R, 3 R)$-3-phenyl-2-oxiranemethanol was completely hydrolyzed. The residue was separated by preparative HPLC $\left(\mathrm{MeOH} / \mathrm{H}_{2} \mathrm{O}, 25: 75, \mathrm{v} \backslash \mathrm{v}, \mathrm{HOAc}, 0.1 \%\right)$ to
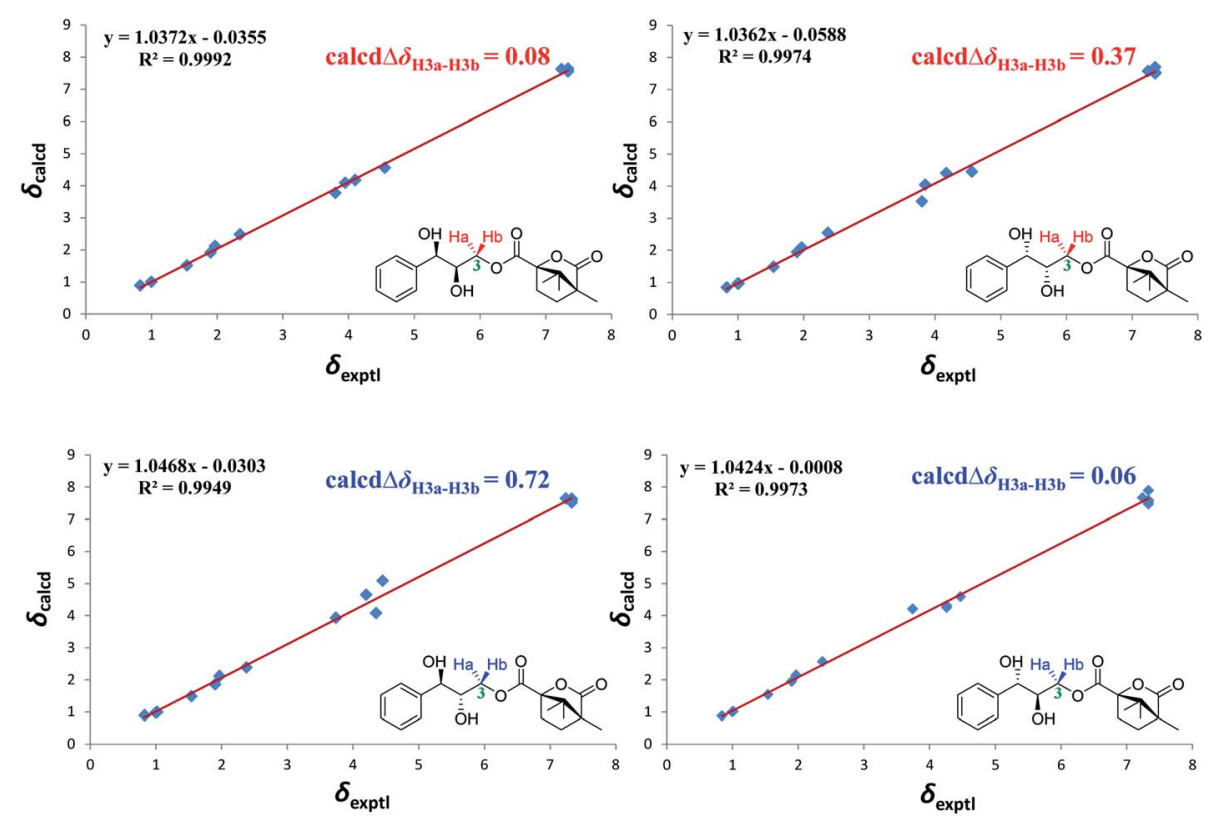

Fig. 6 Linear correlation plots of predicted versus experimental ${ }^{1} \mathrm{H}$ NMR chemical shifts. 
afford M1 (45 mg) and M4 (50 mg). The hydrolysis procedure of $(2 S, 3 S)$-3-phenyl-2-oxiranemethanol was same as the above. The residue was separated by preparative HPLC $\left(\mathrm{MeOH} / \mathrm{H}_{2} \mathrm{O}\right.$, $25:$ : 75, v $\mid \mathrm{v}, \mathrm{HOAc}, 0.1 \%)$ to afford $\mathbf{M} 2$ and M3. Compounds M1M4 are known compounds and they were identified by comparing their spectral data with those of the known compounds. $^{22}$

$(1 R, 2 R)-1-P h e n y l p r o p a n e-1,2,3-t r i o l \quad$ and $\quad(1 S, 2 S)-1-$ phenylpropane-1,2,3-triol (M1 and M2). Colorless oil; the specific rotations were $[\alpha]_{\mathrm{D}}^{20}-39(c 0.1$ methanol $)$ and $[\alpha]_{\mathrm{D}}^{20}+33(c$ 0.1 methanol), respectively. ${ }^{1} \mathrm{H}-\mathrm{NMR}\left(500 \mathrm{MHz}\right.$, methanol- $\left.d_{4}\right)$ : $\delta$ 7.25-7.40 (5H, one mono-substituted benzene ring system), $4.63(1 \mathrm{H}, \mathrm{d}, J=5.5 \mathrm{~Hz}), 3.69(1 \mathrm{H}, \mathrm{m}), 3.50(1 \mathrm{H}, \mathrm{dd}, J=11.5,4.0$ $\mathrm{Hz}), 3.36(1 \mathrm{H}, \mathrm{dd}, J=11.5,6.0 \mathrm{~Hz}) .{ }^{1} \mathrm{H}-\mathrm{NMR}(500 \mathrm{MHz}$, acetone$\left.d_{6}\right): \delta$ 7.23-7.41 $(5 \mathrm{H}$, one mono-substituted benzene ring system $), 4.68(1 \mathrm{H}, \mathrm{d}, J=5.5 \mathrm{~Hz}), 3.65(1 \mathrm{H}, \mathrm{m}), 3.51(1 \mathrm{H}, \mathrm{dd}, J=$ 11.0, $3.5 \mathrm{~Hz}), 3.39(1 \mathrm{H}, \mathrm{dd}, J=11.5,5.5 \mathrm{~Hz}) .{ }^{1} \mathrm{H}-\mathrm{NMR}(500 \mathrm{MHz}$, pyridine- $\left.d_{5}\right): \delta 7.84(2 \mathrm{H}, \mathrm{d}, J=7.5 \mathrm{~Hz}), 7.40(2 \mathrm{H}, \mathrm{t}, J=7.5 \mathrm{~Hz})$, $7.30(1 \mathrm{H}, \mathrm{t}, J=7.5 \mathrm{~Hz}), 5.39(1 \mathrm{H}, \mathrm{d}, J=5.5 \mathrm{~Hz}), 4.39(1 \mathrm{H}, \mathrm{m}), 4.24$ $(1 \mathrm{H}, \mathrm{dd}, J=11.0,4.5 \mathrm{~Hz}), 4.09(1 \mathrm{H}, \mathrm{dd}, J=11.0,6.0 \mathrm{~Hz}) .{ }^{1} \mathrm{H}-$ NMR (500 MHz, DMSO- $\left.d_{6}\right): \delta$ 7.19-7.34 $(5 \mathrm{H}$, one monosubstituted benzene ring system), $4.53(1 \mathrm{H}, \mathrm{t}, J=5.0 \mathrm{~Hz}), 3.48$ $(1 \mathrm{H}, \mathrm{m}), 3.36(1 \mathrm{H}, \mathrm{m}), 3.15(1 \mathrm{H}, \mathrm{m}) .{ }^{1} \mathrm{H}-\mathrm{NMR}\left(500 \mathrm{MHz}, \mathrm{CDCl}_{3}\right)$ : $\delta$ 7.28-7.33 (5H, one mono-substituted benzene ring system), $4.66(1 \mathrm{H}, \mathrm{d}, J=5.5 \mathrm{~Hz}), 3.76(1 \mathrm{H}, \mathrm{brs}), 3.54(1 \mathrm{H}, \mathrm{brd}, J=11.0$ $\mathrm{Hz}), 3.46(1 \mathrm{H}$, brd, $J=11.0 \mathrm{~Hz}) .{ }^{13} \mathrm{C}-\mathrm{NMR}\left(125 \mathrm{MHz}, \mathrm{CDCl}_{3}\right)$ : $\delta$ 140.6, 128.6, 128.6, 128.2, 126.8, 126.8, 76.2, 74.8, 63.2; HRESIMS refer to compounds M3 and M4.

$(1 R, 2 S)$-1-Phenylpropane-1,2,3-triol and $(1 S, 2 R)-1$ phenylpropane-1,2,3-triol (M3 and M4). Colorless oil; the specific rotations were $[\alpha]_{\mathrm{D}}^{20}-47\left(c 0.1\right.$ methanol) and $[\alpha]_{\mathrm{D}}^{20}+27(c$ 0.1 methanol), respectively. ${ }^{1} \mathrm{H}-\mathrm{NMR}\left(500 \mathrm{MHz}\right.$, methanol- $\left.d_{4}\right)$ : $\delta$ 7.24-7.41 (5H, one mono-substituted benzene ring system), $4.61(1 \mathrm{H}, \mathrm{d}, J=6.5 \mathrm{~Hz}), 3.75(1 \mathrm{H}, \mathrm{m}), 3.66(1 \mathrm{H}, \mathrm{dd}, J=11.5,3.5$ $\mathrm{Hz}), 3.60(1 \mathrm{H}, \mathrm{dd}, J=11.5,6.5 \mathrm{~Hz}) ;{ }^{1} \mathrm{H}-\mathrm{NMR}(500 \mathrm{MHz}$, acetone$\left.d_{6}\right): \delta$ 7.21-7.41 $(5 \mathrm{H}$, one mono-substituted benzene ring system), $4.67(1 \mathrm{H}, \mathrm{d}, J=4.0 \mathrm{~Hz}), 3.73(1 \mathrm{H}, \mathrm{m}), 3.61(2 \mathrm{H}$, overlap). ${ }^{1} \mathrm{H}-\mathrm{NMR}\left(500 \mathrm{MHz}\right.$, pyridine- $\left.d_{5}\right): \delta 7.88(2 \mathrm{H}, \mathrm{d}, J=8.0 \mathrm{~Hz}), 7.40$ $(2 \mathrm{H}, \mathrm{t}, J=8.0 \mathrm{~Hz}), 7.30(1 \mathrm{H}, \mathrm{t}, J=8.0 \mathrm{~Hz}), 5.35(1 \mathrm{H}, \mathrm{d}, J=6.0$ $\mathrm{Hz}), 4.50(1 \mathrm{H}, \mathrm{m}), 4.42\left(2 \mathrm{H}\right.$, overlap). ${ }^{1} \mathrm{H}-\mathrm{NMR}(500 \mathrm{MHz}$, DMSO$\left.d_{6}\right): \delta$ 7.19-7.34 $(5 \mathrm{H}$, one mono-substituted benzene ring system), $4.42(1 \mathrm{H}$, overlap), $3.51(1 \mathrm{H}, \mathrm{m}), 3.44(1 \mathrm{H}, \mathrm{m}), 3.38(1 \mathrm{H}$, m). ${ }^{1} \mathrm{H}$-NMR $\left(500 \mathrm{MHz}, \mathrm{CDCl}_{3}\right): \delta 7.30-7.39(5 \mathrm{H}$, one monosubstituted benzene ring system), $4.89(1 \mathrm{H}, \mathrm{d}, J=5.0 \mathrm{~Hz})$, $3.85(1 \mathrm{H}, \mathrm{m}), 3.74(1 \mathrm{H}, \mathrm{dd}, J=11.0,5.5 \mathrm{~Hz}), 3.67(1 \mathrm{H}, \mathrm{dd}, J=$ 11.0, $3.5 \mathrm{~Hz}) .{ }^{13} \mathrm{C}-\mathrm{NMR}\left(125 \mathrm{MHz}, \mathrm{CDCl}_{3}\right): \delta 140.4,128.8,128.8$, 128.3, 126.3, 126.3, 76.1, 74.6, 63.1; HRESIMS $m / z$ 191.0679 [M + $\mathrm{Na}]^{+}$(calcd for $\mathrm{C}_{9} \mathrm{H}_{12} \mathrm{O}_{3} \mathrm{Na}$ 191.0679).

$(2 R, 3 R)$-2,3-Dihydroxy-3-phenylpropyl(1S,4R)-4,7,7trimethyl-3-oxo-2-oxabicyclo[2.2.1] heptane-1-carboxylate (M1a). Compound $\mathbf{M 1}(10 \mathrm{mg}, 0.06 \mathrm{mmol})$ solution in dry $\mathrm{CH}_{2} \mathrm{Cl}_{2}$ or THF was added to a solution of camphanic acid chloride $(13 \mathrm{mg}$, $0.06 \mathrm{mmol}$ ), EDCI (11.4 mg, $0.06 \mathrm{mmol}$ ) and DMAP ( $3.6 \mathrm{mg}, 0.03$ $\mathrm{mmol})$ at $0{ }^{\circ} \mathrm{C}$ with the protection of argon gas. The reaction was stirred at $0{ }^{\circ} \mathrm{C}$ for $1 \mathrm{~h}$ and was detected by TLC. Then, the reaction was quenched by dropwise addition of $\mathrm{H}_{2} \mathrm{O}$. The suspension was extracted with $\mathrm{CH}_{2} \mathrm{Cl}_{2}$. Removal of the solvent under reduced pressure and purification of the residue by preparative HPLC, eluting with $40 \%$ acetonitrile $/ \mathrm{H}_{2} \mathrm{O}$, gave the M1a $8.7 \mathrm{mg}$. Colorless oil; ${ }^{1} \mathrm{H}-\mathrm{NMR}\left(500 \mathrm{MHz}\right.$, methanol $\left.-d_{4}\right)$ : $\delta$ 7.27-7.42 (5H, one mono-substituted benzene ring system), $4.65(1 \mathrm{H}, \mathrm{d}, J=5.5 \mathrm{~Hz}), 4.21(1 \mathrm{H}, \mathrm{dd}, J=11.5,3.5 \mathrm{~Hz}), 4.06(1 \mathrm{H}$, $\mathrm{dd}, J=11.5,7.0 \mathrm{~Hz}), 3.95(1 \mathrm{H}, \mathrm{m}), 2.47(1 \mathrm{H}, \mathrm{m}), 2.03(1 \mathrm{H}, \mathrm{m})$, $1.96(1 \mathrm{H}, \mathrm{m}), 1.62(1 \mathrm{H}, \mathrm{m}), 1.09(3 \mathrm{H}, \mathrm{s}), 1.09(3 \mathrm{H}, \mathrm{s}), 0.94(3 \mathrm{H}, \mathrm{s})$. ${ }^{1} \mathrm{H}-\mathrm{NMR}\left(500 \mathrm{MHz}\right.$, DMSO- $d_{6}$ ): $\delta$ 7.23-7.38 (5H, one monosubstituted benzene ring system), $4.56(1 \mathrm{H}, \mathrm{t}, J=5.0 \mathrm{~Hz}), 4.10$ $(1 \mathrm{H}, \mathrm{dd}, J=11.0,3.5 \mathrm{~Hz}), 3.95(1 \mathrm{H}, \mathrm{dd}, J=11.0,7.5 \mathrm{~Hz}), 3.80$ $(1 \mathrm{H}, \mathrm{m}), 2.35(1 \mathrm{H}, \mathrm{m}), 1.97(1 \mathrm{H}, \mathrm{m}), 1.90(1 \mathrm{H}, \mathrm{m}), 1.54(1 \mathrm{H}, \mathrm{m})$, $1.01(3 \mathrm{H}, \mathrm{s}), 1.00(3 \mathrm{H}, \mathrm{s}), 0.83(3 \mathrm{H}, \mathrm{s}) .{ }^{1} \mathrm{H}-\mathrm{NMR}(500 \mathrm{MHz}$, acetone- $\left.d_{6}\right): \delta$ 7.26-7.45 (5H, one mono-substituted benzene ring system), $4.73(1 \mathrm{H}, \mathrm{t}, J=5.0 \mathrm{~Hz}), 4.24(1 \mathrm{H}, \mathrm{dd}, J=11.5,3.5$ $\mathrm{Hz}), 4.11(1 \mathrm{H}, \mathrm{dd}, J=11.5,7.0 \mathrm{~Hz}), 3.98(1 \mathrm{H}, \mathrm{m}), 2.46(1 \mathrm{H}, \mathrm{m})$, $2.01(1 \mathrm{H}, \mathrm{m}), 1.95(1 \mathrm{H}, \mathrm{m}), 1.60(1 \mathrm{H}, \mathrm{m}), 1.09(3 \mathrm{H}, \mathrm{s}), 1.05(3 \mathrm{H}$, s), $0.92(3 \mathrm{H}, \mathrm{s}) .{ }^{1} \mathrm{H}-\mathrm{NMR}\left(500 \mathrm{MHz}\right.$, pyridine- $\left.d_{5}\right): \delta 7.80(2 \mathrm{H}, \mathrm{d}, J$ $=7.5 \mathrm{~Hz}), 7.42(2 \mathrm{H}, \mathrm{d}, J=7.5 \mathrm{~Hz}), 7.32(1 \mathrm{H}, \mathrm{t}, J=7.5 \mathrm{~Hz}), 5.22$ $(1 \mathrm{H}, \mathrm{d}, J=5.0 \mathrm{~Hz}), 4.75(1 \mathrm{H}, \mathrm{dd}, J=11.0,7.5 \mathrm{~Hz}), 4.70(1 \mathrm{H}, \mathrm{dd}, J$ $=11.0,4.0 \mathrm{~Hz}), 4.52(1 \mathrm{H}, \mathrm{m}), 2.47(1 \mathrm{H}, \mathrm{m}), 1.99(1 \mathrm{H}, \mathrm{m}), 1.82$ $(1 \mathrm{H}, \mathrm{m}), 1.57(1 \mathrm{H}, \mathrm{m}), 1.06(3 \mathrm{H}, \mathrm{s}), 1.05(3 \mathrm{H}, \mathrm{s}), 1.02(3 \mathrm{H}, \mathrm{s}) .{ }^{1} \mathrm{H}-$ $\operatorname{NMR}\left(500 \mathrm{MHz}, \mathrm{CDCl}_{3}\right): \delta 7.30-7.37(5 \mathrm{H}$, one mono-substituted benzene ring system), $4.68(1 \mathrm{H}, \mathrm{d}, J=7.0 \mathrm{~Hz}), 4.25(1 \mathrm{H}, \mathrm{dd}, J=$ $11.5,3.5 \mathrm{~Hz}), 4.11(1 \mathrm{H}, \mathrm{dd}, J=11.5,6.5 \mathrm{~Hz}), 3.98(1 \mathrm{H}, \mathrm{m}), 2.41$ $(1 \mathrm{H}, \mathrm{m}), 2.02(1 \mathrm{H}, \mathrm{m}), 1.92(1 \mathrm{H}, \mathrm{m}), 1.69(1 \mathrm{H}, \mathrm{m}), 1.11(3 \mathrm{H}, \mathrm{s})$, $1.05(3 \mathrm{H}, \mathrm{s}), 0.96(3 \mathrm{H}, \mathrm{s}) .{ }^{13} \mathrm{C}-\mathrm{NMR}\left(125 \mathrm{MHz}\right.$, methanol- $\left.d_{4}\right)$ : $\delta$ 180.3, 168.7, 142.9, 129.3, 129.3, 128.8, 127.9, 127.9, 92.9, 75.6, 74.5, 67.6, 56.0, 55.4, 31.5, 29.9, 17.1, 17.0, 9.9; HRESIMS $\mathrm{m} / \mathrm{z}$ $371.1465[\mathrm{M}+\mathrm{Na}]^{+}$(calcd for $\mathrm{C}_{19} \mathrm{H}_{24} \mathrm{O}_{6} \mathrm{Na} 371.1458$ ).

(2S,3S)-2,3-Dihydroxy-3-phenylpropyl(1S,4R)-4,7,7-trimethyl3-oxo-2-oxabicyclo[2.2.1] heptane-1-carboxylate (M2a). The synthesis procedure of M2a was same with the M1a. Colorless oil; ${ }^{1} \mathrm{H}-\mathrm{NMR}\left(500 \mathrm{MHz}\right.$, methanol- $\left.d_{4}\right): \delta 7.27-7.41(5 \mathrm{H}$, one mono-substituted benzene ring system), $4.67(1 \mathrm{H}, \mathrm{d}, J=6.0 \mathrm{~Hz})$, $4.27(1 \mathrm{H}, \mathrm{dd}, J=11.0,3.5 \mathrm{~Hz}), 3.99(1 \mathrm{H}, \mathrm{dd}, J=11.0,6.5 \mathrm{~Hz})$, $3.94(1 \mathrm{H}, \mathrm{m}), 2.49(1 \mathrm{H}, \mathrm{m}), 2.04(1 \mathrm{H}, \mathrm{m}), 1.97(1 \mathrm{H}, \mathrm{m}), 1.63(1 \mathrm{H}$, $\mathrm{m}), 1.11(3 \mathrm{H}, \mathrm{s}), 1.09(3 \mathrm{H}, \mathrm{s}), 0.94(3 \mathrm{H}, \mathrm{s}) .{ }^{1} \mathrm{H}-\mathrm{NMR}(500 \mathrm{MHz}$, DMSO- $\left.d_{6}\right): \delta 7.23-7.37(5 \mathrm{H}$, one mono-substituted benzene ring system), $4.56(1 \mathrm{H}, \mathrm{d}, J=5.0 \mathrm{~Hz}), 4.18(1 \mathrm{H}, \mathrm{dd}, J=11.0,3.5 \mathrm{~Hz})$, $3.85(1 \mathrm{H}, \mathrm{dd}, J=11.0,7.0 \mathrm{~Hz}), 3.80(1 \mathrm{H}, \mathrm{m}), 2.38(1 \mathrm{H}, \mathrm{m}), 1.97$ $(1 \mathrm{H}, \mathrm{m}), 1.90(1 \mathrm{H}, \mathrm{m}), 1.54(1 \mathrm{H}, \mathrm{m}), 1.01(3 \mathrm{H}, \mathrm{s}), 1.00(3 \mathrm{H}, \mathrm{s})$, $0.83(3 \mathrm{H}, \mathrm{s}) .{ }^{1} \mathrm{H}-\mathrm{NMR}\left(500 \mathrm{MHz}\right.$, acetone- $\left.d_{6}\right): \delta 7.25-7.45(5 \mathrm{H}$, one mono-substituted benzene ring system), $4.73(1 \mathrm{H}, \mathrm{d}, J=5.5$ $\mathrm{Hz}), 4.29(1 \mathrm{H}, \mathrm{dd}, J=11.0,4.0 \mathrm{~Hz}), 4.03(1 \mathrm{H}, \mathrm{dd}, J=11.0,6.5$ $\mathrm{Hz}), 3.97(1 \mathrm{H}, \mathrm{m}), 2.49(1 \mathrm{H}, \mathrm{m}), 2.02(1 \mathrm{H}, \mathrm{m}), 1.96(1 \mathrm{H}, \mathrm{m}), 1.60$ $(1 \mathrm{H}, \mathrm{m}), 1.10(3 \mathrm{H}, \mathrm{s}), 1.05(3 \mathrm{H}, \mathrm{s}), 0.92(3 \mathrm{H}, \mathrm{s}) .{ }^{1} \mathrm{H}-\mathrm{NMR}(500$ MHz, pyridine- $\left.d_{5}\right): \delta 7.80(2 \mathrm{H}, \mathrm{d}, J=8.0 \mathrm{~Hz}), 7.42(2 \mathrm{H}, \mathrm{d}, J=8.0$ $\mathrm{Hz}), 7.32(1 \mathrm{H}, \mathrm{t}, J=8.0 \mathrm{~Hz}), 5.24(1 \mathrm{H}, \mathrm{d}, J=5.0 \mathrm{~Hz}), 4.80(1 \mathrm{H}, \mathrm{dd}$, $J=11.0,3.0 \mathrm{~Hz}), 4.62(1 \mathrm{H}, \mathrm{dd}, J=11.0,7.0 \mathrm{~Hz}), 4.53(1 \mathrm{H}, \mathrm{m})$, $2.50(1 \mathrm{H}, \mathrm{m}), 2.01(1 \mathrm{H}, \mathrm{m}), 1.84(1 \mathrm{H}, \mathrm{m}), 1.58(1 \mathrm{H}, \mathrm{m}), 1.06(3 \mathrm{H}$, $\mathrm{s}), 1.06(3 \mathrm{H}, \mathrm{s}), 1.02(3 \mathrm{H}, \mathrm{s}) .{ }^{1} \mathrm{H}-\mathrm{NMR}\left(500 \mathrm{MHz}, \mathrm{CDCl}_{3}\right): \delta 7.30-$ $7.38(5 \mathrm{H}$, one mono-substituted benzene ring system $), 4.68(1 \mathrm{H}$, $\mathrm{d}, J=6.5 \mathrm{~Hz}), 4.25(1 \mathrm{H}, \mathrm{dd}, J=11.5,3.5 \mathrm{~Hz}), 4.08(1 \mathrm{H}, \mathrm{dd}, J=$ 11.5, $6.5 \mathrm{~Hz}), 3.98(1 \mathrm{H}, \mathrm{m}), 2.42(1 \mathrm{H}, \mathrm{m}), 2.02(1 \mathrm{H}, \mathrm{m}), 1.92(1 \mathrm{H}$, $\mathrm{m}), 1.68(1 \mathrm{H}, \mathrm{m}), 1.11(3 \mathrm{H}, \mathrm{s}), 1.06(3 \mathrm{H}, \mathrm{s}), 0.97(3 \mathrm{H}, \mathrm{s}) .{ }^{13} \mathrm{C}-\mathrm{NMR}$ (125 MHz, methanol- $d_{4}$ ): $\delta 180.3,168.8,142.8,129.3,129.3$, 
128.8, 127.9, 127.9, 92.9, 75.6, 74.5, 67.6, 56.0, 55.4, 31.5, 30.0, 17.1, 17.0, 9.9; HRESIMS refer to compound M1a.

$(2 S, 3 R)-2,3-D i h y d r o x y-3-p h e n y l p r o p y l(1 S, 4 R)-4,7,7-$ trimethyl-3-oxo-2-oxabicyclo[2.2.1] heptane-1-carboxylate (M3a). The synthesis procedure of M3a was same with the M1a. Colorless oil; ${ }^{1} \mathrm{H}-\mathrm{NMR}\left(500 \mathrm{MHz}\right.$, methanol- $\left.d_{4}\right): \delta 7.26-7.42(5 \mathrm{H}$, one mono-substituted benzene ring system), $4.61(1 \mathrm{H}, \mathrm{d}, J=6.5$ $\mathrm{Hz}), 4.42(1 \mathrm{H}, \mathrm{dd}, J=11.5,3.0 \mathrm{~Hz}), 4.32(1 \mathrm{H}, \mathrm{dd}, J=11.5,7.0$ $\mathrm{Hz}), 3.95$ (1H, m), 2.49 (1H, m), 2.04 (1H, m), 1.97 (1H, m), 1.62 (1H, m), 1.10 (3H, s), 1.09 (3H, s), 0.94 (3H, s). ${ }^{1} \mathrm{H}-\mathrm{NMR}(500$ MHz, DMSO- $\left.d_{6}\right): \delta$ 7.23-7.37 (5H, one mono-substituted benzene ring system), $4.46(1 \mathrm{H}, \mathrm{dd}, J=6.5,4.5 \mathrm{~Hz}), 4.35(1 \mathrm{H}$, $\mathrm{dd}, J=11.5,3.0 \mathrm{~Hz}), 4.20(1 \mathrm{H}, \mathrm{dd}, J=11.5,7.0 \mathrm{~Hz}), 3.74(1 \mathrm{H}, \mathrm{m})$, $2.39(1 \mathrm{H}, \mathrm{m}), 1.98(1 \mathrm{H}, \mathrm{m}), 1.90(1 \mathrm{H}, \mathrm{m}), 1.55(1 \mathrm{H}, \mathrm{m}), 1.03(3 \mathrm{H}$, s), $1.00(3 \mathrm{H}, \mathrm{s}), 0.83(3 \mathrm{H}, \mathrm{s}) .{ }^{1} \mathrm{H}-\mathrm{NMR}\left(500 \mathrm{MHz}\right.$, acetone- $\left.d_{6}\right)$ : $\delta$ 7.24-7.44 (5H, one mono-substituted benzene ring system), $4.72(1 \mathrm{H}, \mathrm{dd}, J=6.5,4.5 \mathrm{~Hz}), 4.39(1 \mathrm{H}, \mathrm{dd}, J=11.5,3.0 \mathrm{~Hz}), 4.33$ $(1 \mathrm{H}, \mathrm{dd}, J=11.5,7.0 \mathrm{~Hz}), 4.01(1 \mathrm{H}, \mathrm{m}), 2.48(1 \mathrm{H}, \mathrm{m}), 2.00(1 \mathrm{H}$, m), $1.94(1 \mathrm{H}, \mathrm{m}), 1.59(1 \mathrm{H}, \mathrm{m}), 1.09(3 \mathrm{H}, \mathrm{s}), 1.05(3 \mathrm{H}, \mathrm{s}), 0.92$ $(3 \mathrm{H}, \mathrm{s}) .{ }^{1} \mathrm{H}-\mathrm{NMR}\left(500 \mathrm{MHz}\right.$, pyridine- $\left.d_{5}\right): \delta 7.82(2 \mathrm{H}, \mathrm{d}, J=7.5$ $\mathrm{Hz}), 7.41(2 \mathrm{H}, \mathrm{d}, J=7.5 \mathrm{~Hz}), 7.31(1 \mathrm{H}, \mathrm{t}, J=7.5 \mathrm{~Hz}), 5.22(1 \mathrm{H}, \mathrm{d}, J$ $=6.5 \mathrm{~Hz}), 5.06(1 \mathrm{H}, \mathrm{dd}, J=11.5,3.0 \mathrm{~Hz}), 4.98(1 \mathrm{H}, \mathrm{dd}, J=11.5$, $7.0 \mathrm{~Hz}), 4.55(1 \mathrm{H}, \mathrm{m}), 2.49(1 \mathrm{H}, \mathrm{m}), 1.99(1 \mathrm{H}, \mathrm{m}), 1.82(1 \mathrm{H}, \mathrm{m})$, $1.57(1 \mathrm{H}, \mathrm{m}), 1.07$ (3H, s), 1.06 (3H, s), 1.05 (3H, s). ${ }^{1} \mathrm{H}-\mathrm{NMR}(500$ $\left.\mathrm{MHz}, \mathrm{CDCl}_{3}\right): \delta$ 7.29-7.38 $(5 \mathrm{H}$, one mono-substituted benzene ring system), $4.83(1 \mathrm{H}, \mathrm{d}, J=5.5 \mathrm{~Hz}), 4.34(1 \mathrm{H}, \mathrm{dd}, J=11.5,7.5$ $\mathrm{Hz}), 4.25(1 \mathrm{H}, \mathrm{dd}, J=11.5,3.0 \mathrm{~Hz}), 4.09(1 \mathrm{H}, \mathrm{m}), 2.40(1 \mathrm{H}, \mathrm{m})$, $2.00(1 \mathrm{H}, \mathrm{m}), 1.92(1 \mathrm{H}, \mathrm{m}), 1.67(1 \mathrm{H}, \mathrm{m}), 1.10(3 \mathrm{H}, \mathrm{s}), 1.04(3 \mathrm{H}$, s), $0.95(3 \mathrm{H}, \mathrm{s}) .{ }^{13} \mathrm{C}-\mathrm{NMR}\left(125 \mathrm{MHz}\right.$, methanol- $\left.d_{4}\right): \delta 180.3,168.9$, 143.2, 129.2, 129.2, 128.6, 128.1, 128.1, 93.0, 75.8, 74.1, 67.8, $56.0,55.4,31.5,30.0,17.1,17.0,9.9$; HRESIMS refer to compound M1a.

$(2 R, 3 S)-2,3-D i h y d r o x y-3-p h e n y l p r o p y l(1 S, 4 R)-4,7,7-$ trimethyl-3-oxo-2-oxabicyclo[2.2.1] heptane-1-carboxylate (M4a). The synthesis procedure of M4a was same with the M1a. Colorless oil; ${ }^{1} \mathrm{H}-\mathrm{NMR}\left(500 \mathrm{MHz}\right.$, methanol- $\left.d_{4}\right): \delta 7.26-7.42(5 \mathrm{H}$, one mono-substituted benzene ring system), $4.62(1 \mathrm{H}, \mathrm{d}, J=7.0$ $\mathrm{Hz}), 4.38(1 \mathrm{H}, \mathrm{dd}, J=11.5,6.5 \mathrm{~Hz}), 4.35(1 \mathrm{H}, \mathrm{dd}, J=11.5,3.5$ $\mathrm{Hz}), 3.96$ (1H, m), 2.49 (1H, m), 2.03 (1H, m), 1.97 (1H, m), 1.62 (1H, m), 1.10 (3H, s), 1.09 (3H, s), 0.94 (3H, s). ${ }^{1} \mathrm{H}-\mathrm{NMR}$ (500 $\left.\mathrm{MHz}, \quad \mathrm{DMSO}-d_{6}\right): \delta 7.22-7.36(5 \mathrm{H}$, one mono-substituted benzene ring system), $4.47(1 \mathrm{H}, \mathrm{dd}, J=6.5,4.5 \mathrm{~Hz}), 4.26(2 \mathrm{H}$, overlap), $3.75(1 \mathrm{H}, \mathrm{m}), 2.38(1 \mathrm{H}, \mathrm{m}), 1.97(1 \mathrm{H}, \mathrm{m}), 1.90(1 \mathrm{H}, \mathrm{m})$, $1.54(1 \mathrm{H}, \mathrm{m}), 1.01(3 \mathrm{H}, \mathrm{s}), 1.00$ (3H, s), 0.84 (3H, s). ${ }^{1} \mathrm{H}-\mathrm{NMR}(500$ $\mathrm{MHz}$, acetone- $\left.d_{6}\right): \delta 7.24-7.44(5 \mathrm{H}$, one mono-substituted benzene ring system), $4.72(1 \mathrm{H}, \mathrm{dd}, J=6.0,4.5 \mathrm{~Hz}), 4.38(1 \mathrm{H}$, $\mathrm{dd}, J=11.5,7.0 \mathrm{~Hz}), 4.33(1 \mathrm{H}, \mathrm{dd}, J=11.5,3.5 \mathrm{~Hz}), 4.01(1 \mathrm{H}, \mathrm{m})$, $2.47(1 \mathrm{H}, \mathrm{m}), 2.00(1 \mathrm{H}, \mathrm{m}), 1.94(1 \mathrm{H}, \mathrm{m}), 1.59(1 \mathrm{H}, \mathrm{m}), 1.09(3 \mathrm{H}$, s), $1.05(3 \mathrm{H}, \mathrm{s}), 0.92(3 \mathrm{H}, \mathrm{s}) .{ }^{1} \mathrm{H}-\mathrm{NMR}\left(500 \mathrm{MHz}\right.$, pyridine- $\left.d_{5}\right)$ : $\delta 7.82(2 \mathrm{H}, \mathrm{d}, J=7.5 \mathrm{~Hz}), 7.41(2 \mathrm{H}, \mathrm{d}, J=7.5 \mathrm{~Hz}), 7.31(1 \mathrm{H}, \mathrm{t}, J=$ $7.5 \mathrm{~Hz}), 5.22(1 \mathrm{H}, \mathrm{d}, J=7.0 \mathrm{~Hz}), 5.06(1 \mathrm{H}, \mathrm{dd}, J=11.5,7.0 \mathrm{~Hz})$, $4.98(1 \mathrm{H}, \mathrm{dd}, J=11.5,3.0 \mathrm{~Hz}), 4.55(1 \mathrm{H}, \mathrm{m}), 2.50(1 \mathrm{H}, \mathrm{m}), 1.99$ $(1 \mathrm{H}, \mathrm{m}), 1.83(1 \mathrm{H}, \mathrm{m}), 1.57(1 \mathrm{H}, \mathrm{m}), 1.07(3 \mathrm{H}, \mathrm{s}), 1.06(3 \mathrm{H}, \mathrm{s})$, $1.04(3 \mathrm{H}, \mathrm{s}) .{ }^{1} \mathrm{H}-\mathrm{NMR}\left(500 \mathrm{MHz}, \mathrm{CDCl}_{3}\right): \delta 7.30-7.38(5 \mathrm{H}$, one mono-substituted benzene ring system), $4.84(1 \mathrm{H}, \mathrm{d}, J=5.5 \mathrm{~Hz})$, $4.38(1 \mathrm{H}, \mathrm{dd}, J=11.5,7.5 \mathrm{~Hz}), 4.24(1 \mathrm{H}, \mathrm{dd}, J=11.5,3.0 \mathrm{~Hz})$, $4.08(1 \mathrm{H}, \mathrm{m}), 2.41(1 \mathrm{H}, \mathrm{m}), 2.01(1 \mathrm{H}, \mathrm{m}), 1.92(1 \mathrm{H}, \mathrm{m}), 1.68(1 \mathrm{H}$, m), 1.11 (3H, s), 1.04 (3H, s), 0.95 (3H, s). ${ }^{13} \mathrm{C}-\mathrm{NMR}(125 \mathrm{MHz}$, methanol- $\left.d_{4}\right): \delta 180.3,168.9,143.2,129.2,129.2,128.6,128.1$, 128.1, 93.0, 75.8, 74.2 , 67.8, 56.0, 55.4, 31.5, 30.0, 17.1, 17.0, 9.9; HRESIMS refer to compound M1a.

$(1 R, 2 R)-1-(4-H y d r o x y-3,5-d i m e t h o x y p h e n y l) p r o p a n e-1,2,3-$ triol and (1S,2S)-1-(4-hydroxy-3,5-dimethoxyphenyl)propane1,2,3-triol (1 and 2). Colorless oil; the specific rotations were

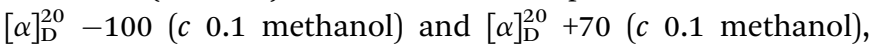
respectively. ${ }^{1} \mathrm{H}-\mathrm{NMR}\left(500 \mathrm{MHz}\right.$, methanol- $\left.d_{4}\right): \delta 6.68(2 \mathrm{H}, \mathrm{brs})$, $4.53(1 \mathrm{H}, \mathrm{d}, J=6.0 \mathrm{~Hz}), 3.67(1 \mathrm{H}, \mathrm{m}), 3.50$ (1H, dd, $J=11.5,4.0$ $\mathrm{Hz}), 3.37(1 \mathrm{H}, \mathrm{dd}, J=11.5,6.5 \mathrm{~Hz}) .{ }^{1} \mathrm{H}-\mathrm{NMR}(500 \mathrm{MHz}$, acetone$d 6): \delta 6.69$ (2H, brs), $4.56(1 \mathrm{H}, \mathrm{d}, J=6.0 \mathrm{~Hz}), 3.62(1 \mathrm{H}, \mathrm{m}), 3.49$ $(1 \mathrm{H}, \mathrm{dd}, J=11.0,4.0 \mathrm{~Hz}), 3.40(1 \mathrm{H}, \mathrm{dd}, J=11.0,6.0 \mathrm{~Hz}) \cdot{ }^{13} \mathrm{C}-$ NMR (125 MHz, methanol- $\left.d_{4}\right): \delta$ 149.1, 149.1, 135.9, 134.1, 105.0, 105.0, 77.6, 75.6, 64.2, 56.7, 56.7; HRESIMS $m / z 267.0839$ $[\mathrm{M}+\mathrm{Na}]^{+}$(calcd for $\mathrm{C}_{11} \mathrm{H}_{16} \mathrm{O}_{6} \mathrm{Na} 267.0837$ ). Compounds 1 and 2 are known compounds and they were identified by comparing their spectral data with those of the known compounds. ${ }^{8}$

4-((1R,2R)-1,2-Dihydroxy-3-(((1S,4R)-4,7,7-trimethyl-3-oxo-2oxabicyclo[2.2.1] heptane-1-carbonyl)oxy)propyl)-2,6-dimethoxyphenyl $(1 S, 4 R)-4,7,7$-trimethyl-3-oxo-2-oxabicyclo[2.2.1]

heptane-1-carboxylate (1a). Compound 1 (6 $\mathrm{mg}, 0.02 \mathrm{mmol}$ ) solution in dry $\mathrm{CH}_{2} \mathrm{Cl}_{2}$ or THF was added to a solution of camphanic acid chloride ( $9 \mathrm{mg}, 0.04 \mathrm{mmol}$ ), EDCI $(7.6 \mathrm{mg}, 0.04$ mmol) and DMAP $(2.4 \mathrm{mg}, 0.02 \mathrm{mmol})$ at $0{ }^{\circ} \mathrm{C}$ with the protection of argon gas. The reaction was stirred at $0{ }^{\circ} \mathrm{C}$ for $1 \mathrm{~h}$ and was detected by TLC. Then, the reaction was quenched by dropwise addition of $\mathrm{H}_{2} \mathrm{O}$. The suspension was extracted with $\mathrm{CH}_{2} \mathrm{Cl}_{2}$. Removal of the solvent under reduced pressure and purification of the residue by preparative HPLC, eluting with $40 \%$ acetonitrile $/ \mathrm{H}_{2} \mathrm{O}$, gave the $1 \mathrm{a} 2.9 \mathrm{mg}$. Colorless oil; ${ }^{1} \mathrm{H}-\mathrm{NMR}$ (500 MHz, methanol- $\left.d_{4}\right): \delta 6.84(2 \mathrm{H}, \mathrm{brs}), 4.68(1 \mathrm{H}, \mathrm{d}, J=5.0$ $\mathrm{Hz}), 4.33(1 \mathrm{H}, \mathrm{dd}, J=11.5,4.0 \mathrm{~Hz}), 4.16(1 \mathrm{H}, \mathrm{dd}, J=11.5,6.5$ $\mathrm{Hz}), 3.97(1 \mathrm{H}, \mathrm{m}), 2.67(1 \mathrm{H}, \mathrm{m}), 2.48(1 \mathrm{H}, \mathrm{m}), 2.12$ (2H, overlap), $2.04(1 \mathrm{H}, \mathrm{m}), 1.97(1 \mathrm{H}, \mathrm{m}), 1.70(1 \mathrm{H}, \mathrm{m}), 1.63(1 \mathrm{H}, \mathrm{m}), 1.22(3 \mathrm{H}$, s), $1.14(3 \mathrm{H}, \mathrm{s}), 1.10(3 \mathrm{H}, \mathrm{s}), 1.09(3 \mathrm{H}, \mathrm{s}), 1.08(3 \mathrm{H}, \mathrm{s}), 0.97(3 \mathrm{H}, \mathrm{s})$; HRESIMS $m / z 605.2593[\mathrm{M}+\mathrm{H}]^{+}$(calcd for $\mathrm{C}_{31} \mathrm{H}_{41} \mathrm{O}_{12} 605.2593$ ).

4-((1S,2S)-1,2-Dihydroxy-3-(((1S,4R)-4,7,7-trimethyl-3-oxo-2oxabicyclo[2.2.1] heptane-1-carbonyl)oxy)propyl)-2,6-dimethoxyphenyl $(1 S, 4 R)$-4,7,7-trimethyl-3-oxo-2-oxabicyclo[2.2.1]

heptane-1-carboxylate (2a). Compound $2(4.4 \mathrm{mg}, 0.018 \mathrm{mmol})$ solution in dry $\mathrm{CH}_{2} \mathrm{Cl}_{2}$ or THF was added to a solution of camphanic acid chloride $(7.8 \mathrm{mg}, 0.036 \mathrm{mmol})$, EDCI $(6.8 \mathrm{mg}$, $0.036 \mathrm{mmol})$ and DMAP $(2.2 \mathrm{mg}, 0.018 \mathrm{mmol})$ at $0{ }^{\circ} \mathrm{C}$ with the protection of argon gas. The reaction was stirred at $0{ }^{\circ} \mathrm{C}$ for $1 \mathrm{~h}$ and was detected by TLC. Then, the reaction was quenched by dropwise addition of $\mathrm{H}_{2} \mathrm{O}$. The suspension was extracted with $\mathrm{CH}_{2} \mathrm{Cl}_{2}$. Removal of the solvent under reduced pressure and purification of the residue by preparative HPLC, eluting with $55 \%$ methanol $/ \mathrm{H}_{2} \mathrm{O}$, gave the 2 a $2.0 \mathrm{mg}$. Colorless oil; ${ }^{1} \mathrm{H}-\mathrm{NMR}$ (500 MHz, methanol- $\left.d_{4}\right): \delta 6.82(2 \mathrm{H}, \mathrm{brs}), 4.69(1 \mathrm{H}, \mathrm{d}, J=5.0$ $\mathrm{Hz}), 4.36(1 \mathrm{H}, \mathrm{dd}, J=11.5,4.0 \mathrm{~Hz}), 4.13(1 \mathrm{H}, \mathrm{dd}, J=11.5,6.5$ $\mathrm{Hz}), 3.97(1 \mathrm{H}, \mathrm{m}), 2.67(1 \mathrm{H}, \mathrm{m}), 2.49(1 \mathrm{H}, \mathrm{m}), 2.12$ (2H, overlap), 2.02 (2H, overlap), $1.70(1 \mathrm{H}, \mathrm{m}), 1.63(1 \mathrm{H}, \mathrm{m}), 1.22(3 \mathrm{H}, \mathrm{s}), 1.14$ (3H, s), 1.11 (3H, s), 1.09 (3H, s), 1.08 (3H, s), $0.94(3 \mathrm{H}, \mathrm{s})$; HRESIMS $m / z$ 605.2593 [M + H] $]^{+}$(calcd for $\mathrm{C}_{31} \mathrm{H}_{41} \mathrm{O}_{12} 605.2600$ ). 
((2R,3R)-3-(4-Bromophenyl)oxiran-2-yl)methanol ((2S,3S)-3-(4-bromophenyl)oxiran-2-yl)methanol. The asymmetric epoxidation was also carried out according to the Sharpless asymmetric Epoxidation. Colorless oil; the specific rotations were $[\alpha]_{\mathrm{D}}^{20}+45(c \quad 0.1$ methanol $)$ and $[\alpha]_{\mathrm{D}}^{20}-25\left(\begin{array}{cc}c & 0.1\end{array}\right.$ methanol), respectively. ${ }^{1} \mathrm{H}-\mathrm{NMR}\left(500 \mathrm{MHz}\right.$, methanol- $\left.d_{4}\right)$ : $\delta 7.49(2 \mathrm{H}, \mathrm{d}, J=8.5 \mathrm{~Hz}), 7.22(2 \mathrm{H}, \mathrm{d}, J=8.5 \mathrm{~Hz}), 3.85(1 \mathrm{H}, \mathrm{dd}, J$ $=12.5,3.0 \mathrm{~Hz}), 3.83(1 \mathrm{H}, \mathrm{d}, J=1.5 \mathrm{~Hz}), 3.67(1 \mathrm{H}, \mathrm{dd}, J=12.5$, $4.5 \mathrm{~Hz}$ ), $3.13(1 \mathrm{H}, \mathrm{m}) ;{ }^{13} \mathrm{C}-\mathrm{NMR}\left(125 \mathrm{MHz}\right.$, methanol- $\left.d_{4}\right): \delta 138.2$, 132.6, 132.6, 128.7, 128.7, 122.8, 63.9, 62.6, 56.2; HRESIMS $m / z$ $228.9859[\mathrm{M}+\mathrm{H}]^{+}$(calcd for $\mathrm{C}_{9} \mathrm{H}_{10} \mathrm{O}_{2} \mathrm{Br} 228.9858$ ).

A solution of ((2R,3R)-3-(4-bromophenyl)oxiran-2-yl) methanol (65 mg, $0.3 \mathrm{mmol})$ and acetic acid $(0.5 \mathrm{ml})$ in acetonitrile was stirred by rotary evaporator at $55^{\circ} \mathrm{C}$ until the solution was dry. Through the analysis of HPLC, the $((2 R, 3 R)-3-(4-$ bromophenyl)oxiran-2-yl)methanol was completely hydrolyzed. The residue was separated by preparative $\mathrm{HPLC}\left(\mathrm{MeOH} / \mathrm{H}_{2} \mathrm{O}\right.$, $45: 55, \mathrm{v} \backslash \mathrm{v})$ to afford $3(27 \mathrm{mg})$ and $6(38 \mathrm{mg})$. The hydrolysis procedure of $((2 S, 3 S)$-3-(4-bromophenyl)oxiran-2-yl)methanol was same as the above. The residue was separated by preparative HPLC $\left(\mathrm{MeOH} / \mathrm{H}_{2} \mathrm{O}, 45: 55, \mathrm{v} \backslash \mathrm{v}\right)$ to afford 4 and 5 . Compounds 3-6 are known compounds and their absolute configurations were identified by comparing the optical rotations of M1-M4.

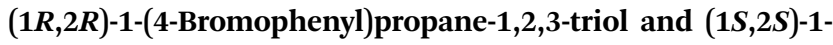
(4-bromophenyl)propane-1,2,3-triol (3 and 4). Colorless oil; the specific rotations were $[\alpha]_{\mathrm{D}}^{20}-20(c 0.1$ methanol $)$ and $[\alpha]_{\mathrm{D}}^{20}+16(c$ 0.1 methanol), respectively. ${ }^{1} \mathrm{H}-\mathrm{NMR}\left(500 \mathrm{MHz}\right.$, methanol- $\left.d_{4}\right)$ : $\delta 7.48(2 \mathrm{H}, \mathrm{d}, J=8.5 \mathrm{~Hz}), 7.33(2 \mathrm{H}, \mathrm{d}, J=8.5 \mathrm{~Hz}), 4.65(1 \mathrm{H}, \mathrm{d}, J=$ $5.0 \mathrm{~Hz}), 3.65(1 \mathrm{H}, \mathrm{m}), 3.55(1 \mathrm{H}, \mathrm{dd}, J=11.5,4.5 \mathrm{~Hz}), 3.39(1 \mathrm{H}$, $\mathrm{dd}, J=11.5,6.5 \mathrm{~Hz}) .{ }^{1} \mathrm{H}-\mathrm{NMR}\left(500 \mathrm{MHz}\right.$, acetone- $\left.d_{6}\right): \delta 7.49(2 \mathrm{H}$, $\mathrm{d}, J=8.5 \mathrm{~Hz}), 7.37(2 \mathrm{H}, \mathrm{d}, J=8.5 \mathrm{~Hz}), 4.72(1 \mathrm{H}, \mathrm{t}, J=4.5 \mathrm{~Hz})$, $3.64(1 \mathrm{H}, \mathrm{m}), 3.56(1 \mathrm{H}, \mathrm{m}), 3.42(1 \mathrm{H}, \mathrm{m}) .{ }^{1} \mathrm{H}-\mathrm{NMR}(500 \mathrm{MHz}$, pyridine- $\left.d_{5}\right): \delta 7.71(2 \mathrm{H}, \mathrm{d}, J=8.5 \mathrm{~Hz}), 7.55(2 \mathrm{H}, \mathrm{d}, J=8.5 \mathrm{~Hz})$, $5.38(1 \mathrm{H}, \mathrm{d}, J=5.0 \mathrm{~Hz}), 4.35(1 \mathrm{H}, \mathrm{m}), 4.28(1 \mathrm{H}, \mathrm{dd}, J=11.0,4.5$ $\mathrm{Hz}), 4.09$ (1H, dd, $J=11.0,6.0 \mathrm{~Hz}) .{ }^{1} \mathrm{H}-\mathrm{NMR}$ (500 MHz, DMSO$\left.d_{6}\right): \delta 7.48(2 \mathrm{H}, \mathrm{d}, J=8.5 \mathrm{~Hz}), 7.29(2 \mathrm{H}, \mathrm{d}, J=8.5 \mathrm{~Hz}), 4.55(1 \mathrm{H}, \mathrm{t}$, $J=5.0 \mathrm{~Hz}), 3.46$ (1H, m), 3.39 (1H, m), $3.15(1 \mathrm{H}, \mathrm{m}) .{ }^{13} \mathrm{C}-\mathrm{NMR}$ (125 MHz, methanol- $d_{4}$ ): $\delta$ 143.0, 132.2, 132.2, 129.8, 129.8, 122.0, 77.1, 74.4, 64.1; HRESIMS $m / z$ 268.9784 [M + Na $]^{+}$(calcd for $\left.\mathrm{C}_{9} \mathrm{H}_{11} \mathrm{O}_{3} \mathrm{BrNa} 268.9778\right)$.

$(1 R, 2 S)$-1-(4-Bromophenyl)propane-1,2,3-triol and $(1 S, 2 R)-1$ (4-bromophenyl)propane-1,2,3-triol (5 and 6). Colorless oil; the specific rotations were $[\alpha]_{\mathrm{D}}^{20}-8\left(c 0.1\right.$ methanol) and $[\alpha]_{\mathrm{D}}^{20}+12(c$ 0.1 methanol), respectively. ${ }^{1} \mathrm{H}-\mathrm{NMR}\left(500 \mathrm{MHz}\right.$, methanol- $\left.d_{4}\right)$ : $\delta 7.48(2 \mathrm{H}, \mathrm{d}, J=8.5 \mathrm{~Hz}), 7.33(2 \mathrm{H}, \mathrm{d}, J=8.5 \mathrm{~Hz}), 4.58(1 \mathrm{H}, \mathrm{d}, J=$ $6.5 \mathrm{~Hz}), 3.70(1 \mathrm{H}, \mathrm{m}), 3.64(1 \mathrm{H}, \mathrm{dd}, J=11.5,4.0 \mathrm{~Hz}), 3.59(1 \mathrm{H}$, $\mathrm{dd}, J=11.5,6.5 \mathrm{~Hz}) .{ }^{1} \mathrm{H}-\mathrm{NMR}\left(500 \mathrm{MHz}\right.$, acetone- $\left.d_{6}\right): \delta 7.48(2 \mathrm{H}$, $\mathrm{d}, J=8.5 \mathrm{~Hz}), 7.37(2 \mathrm{H}, \mathrm{d}, J=8.5 \mathrm{~Hz}), 4.65(1 \mathrm{H}, \mathrm{d}, J=6.5 \mathrm{~Hz})$, 3.69 (1H, m), 3.62 (2H, overlap). ${ }^{1} \mathrm{H}-\mathrm{NMR}(500 \mathrm{MHz}$, pyridine$\left.d_{5}\right): \delta 7.74(2 \mathrm{H}, \mathrm{d}, J=8.5 \mathrm{~Hz}), 7.55(2 \mathrm{H}, \mathrm{d}, J=8.5 \mathrm{~Hz}), 5.30(1 \mathrm{H}$, $\mathrm{dd}, J=5.5,3.0 \mathrm{~Hz}), 4.42(1 \mathrm{H}, \mathrm{m}), 4.39$ (2H, overlap). ${ }^{1} \mathrm{H}-\mathrm{NMR}$ $\left(500 \mathrm{MHz}, \mathrm{DMSO}-d_{6}\right): \delta 7.47(2 \mathrm{H}, \mathrm{d}, J=8.5 \mathrm{~Hz}), 7.28(2 \mathrm{H}, \mathrm{d}, J$ $=8.5 \mathrm{~Hz}), 4.41(1 \mathrm{H}, \mathrm{t}, J=5.0 \mathrm{~Hz}), 3.47(1 \mathrm{H}, \mathrm{m}), 3.42(1 \mathrm{H}, \mathrm{m})$, $3.37(1 \mathrm{H}, \mathrm{m}) .{ }^{13} \mathrm{C}-\mathrm{NMR}\left(125 \mathrm{MHz}\right.$, methanol- $\left.d_{4}\right): \delta$ 142.9, 132.0,
132.0, 130.3, 130.3, 122.0, 76.5, 75.3, 64.3; HRESIMS refer to compounds 3 and 4.

$(2 R, 3 R)-3-(4-B r o m o p h e n y l)-2,3-d i h y d r o x y p r o p y l(1 S, 4 R)-$ 4,7,7-trimethyl-3-oxo-2-oxabicyclo[2.2.1] heptane-1-carboxylate (3a). The synthesis procedure of 3a was same with the M1a. Colorless oil; ${ }^{1} \mathrm{H}-\mathrm{NMR}\left(500 \mathrm{MHz}\right.$, methanol- $\left.d_{4}\right)$ : $\delta 7.50(2 \mathrm{H}, \mathrm{d}, J$ $=8.5 \mathrm{~Hz}), 7.35(2 \mathrm{H}, \mathrm{d}, J=8.5 \mathrm{~Hz}), 4.66(1 \mathrm{H}, \mathrm{d}, J=5.0 \mathrm{~Hz}), 4.26$ $(1 \mathrm{H}, \mathrm{dd}, J=11.5,4.0 \mathrm{~Hz}), 4.11(1 \mathrm{H}, \mathrm{dd}, J=11.5,7.0 \mathrm{~Hz}), 3.92$ $(1 \mathrm{H}, \mathrm{m}), 2.46(1 \mathrm{H}, \mathrm{m}), 2.03(1 \mathrm{H}, \mathrm{m}), 1.97(1 \mathrm{H}, \mathrm{m}), 1.63(1 \mathrm{H}, \mathrm{m})$, 1.09 (6H, overlap), $0.94(3 \mathrm{H}, \mathrm{s}) .{ }^{1} \mathrm{H}-\mathrm{NMR}$ (500 MHz, DMSO- $\left.d_{6}\right)$ : $\delta 7.51(2 \mathrm{H}, \mathrm{d}, J=8.5 \mathrm{~Hz}), 7.31(2 \mathrm{H}, \mathrm{d}, J=8.5 \mathrm{~Hz}), 4.56(1 \mathrm{H}, \mathrm{d}, J=$ $3.5 \mathrm{~Hz}), 4.12(1 \mathrm{H}, \mathrm{dd}, J=11.0,3.5 \mathrm{~Hz}), 3.97(1 \mathrm{H}, \mathrm{dd}, J=11.0,8.0$ $\mathrm{Hz}), 3.79(1 \mathrm{H}, \mathrm{m}), 2.35(1 \mathrm{H}, \mathrm{m}), 1.97(1 \mathrm{H}, \mathrm{m}), 1.90(1 \mathrm{H}, \mathrm{m}), 1.54$ (1H, m), 1.00 (6H, overlap), $0.82(3 \mathrm{H}, \mathrm{s}) .{ }^{13} \mathrm{C}-\mathrm{NMR}(125 \mathrm{MHz}$, methanol- $\left.d_{4}\right): \delta 180.3,168.7,142.4,132.3,132.3,129.9,129.9$, 122.3, 92.9, 74.7, 74.2, 67.6, 56.1, 55.4, 31.5, 29.9, 17.1, 17.0, 9.9; HRESIMS $m / z$ 449.0570 $[\mathrm{M}+\mathrm{Na}]^{+}$(calcd for $\mathrm{C}_{19} \mathrm{H}_{23} \mathrm{O}_{6} \mathrm{BrNa}$ 449.0562).

$(2 S, 3 S)-3-(4-B r o m o p h e n y l)-2,3-d i h y d r o x y p r o p y l(1 S, 4 R)-4,7,7-$ trimethyl-3-oxo-2-oxabicyclo[2.2.1] heptane-1-carboxylate (4a). The synthesis procedure of $4 \mathbf{a}$ was same with the M1a. Colorless oil; ${ }^{1} \mathrm{H}-\mathrm{NMR}$ (500 MHz, methanol- $\left.d_{4}\right): \delta 7.50(2 \mathrm{H}, \mathrm{d}, J=8.5 \mathrm{~Hz})$, $7.34(2 \mathrm{H}, \mathrm{d}, J=8.5 \mathrm{~Hz}), 4.67(1 \mathrm{H}, \mathrm{d}, J=5.0 \mathrm{~Hz}), 4.31(1 \mathrm{H}, \mathrm{dd}, J=$ 11.5, $4.0 \mathrm{~Hz}), 4.04$ (1H, dd, $J=11.5,7.0 \mathrm{~Hz}), 3.91(1 \mathrm{H}, \mathrm{m}), 2.48$ (1H, m), $2.04(1 \mathrm{H}, \mathrm{m}), 1.98(1 \mathrm{H}, \mathrm{m}), 1.63(1 \mathrm{H}, \mathrm{m}), 1.10(3 \mathrm{H}, \mathrm{s})$, $1.09(3 \mathrm{H}, \mathrm{s}), 0.93(3 \mathrm{H}, \mathrm{s}) .{ }^{1} \mathrm{H}-\mathrm{NMR}\left(500 \mathrm{MHz}, \mathrm{DMSO}-d_{6}\right): \delta 7.51$ $(2 \mathrm{H}, \mathrm{d}, J=8.0 \mathrm{~Hz}), 7.31(2 \mathrm{H}, \mathrm{d}, J=8.0 \mathrm{~Hz}), 4.56(1 \mathrm{H}, \mathrm{d}, J=3.5$ $\mathrm{Hz}), 4.19$ (1H, dd, $J=11.5,3.5 \mathrm{~Hz}), 3.88(1 \mathrm{H}, \mathrm{dd}, J=11.5,7.0$ $\mathrm{Hz}), 3.79$ (1H, m), 2.36 (1H, m), 1.97 (1H, m), 1.90 (1H, m), 1.54 (1H, m), 1.00 (6H, overlap), $0.82(3 \mathrm{H}, \mathrm{s}) .{ }^{13} \mathrm{C}-\mathrm{NMR}(125 \mathrm{MHz}$, methanol- $\left.d_{4}\right): \delta 180.3,168.7,142.4,132.3,132.3,129.9,129.9$, 122.3, 92.9, 74.7, 74.1, 67.5, 56.0, 55.4, 31.5, 30.0, 17.1, 17.0, 9.9; HRESIMS refer to compounds $3 \mathbf{a}$.

(2S,3R)-3-(4-Bromophenyl)-2,3-dihydroxypropyl(1S,4R)-4,7,7trimethyl-3-oxo-2-oxabicyclo[2.2.1] heptane-1-carboxylate (5a). The synthesis procedure of 5a was same with the M1a. Colorless oil; ${ }^{1} \mathrm{H}-\mathrm{NMR}$ (500 MHz, methanol- $\left.d_{4}\right): \delta 7.50(2 \mathrm{H}, \mathrm{d}, J=8.5 \mathrm{~Hz})$, $7.34(2 \mathrm{H}, \mathrm{d}, J=8.5 \mathrm{~Hz}), 4.57(1 \mathrm{H}, \mathrm{d}, J=7.0 \mathrm{~Hz}), 4.42(1 \mathrm{H}, \mathrm{dd}, J=$ $11.5,3.5 \mathrm{~Hz}), 4.31(1 \mathrm{H}, \mathrm{dd}, J=11.5,7.0 \mathrm{~Hz}), 3.89(1 \mathrm{H}, \mathrm{m}), 2.48$ $(1 \mathrm{H}, \mathrm{m}), 2.03(1 \mathrm{H}, \mathrm{m}), 1.98(1 \mathrm{H}, \mathrm{m}), 1.63(1 \mathrm{H}, \mathrm{m}), 1.10(3 \mathrm{H}, \mathrm{s})$, $1.09(3 \mathrm{H}, \mathrm{s}), 0.94(3 \mathrm{H}, \mathrm{s}) .{ }^{1} \mathrm{H}-\mathrm{NMR}\left(500 \mathrm{MHz}, \mathrm{DMSO}-d_{6}\right): \delta 7.51$ $(2 \mathrm{H}, \mathrm{d}, J=8.5 \mathrm{~Hz}), 7.30(2 \mathrm{H}, \mathrm{d}, J=8.5 \mathrm{~Hz}), 4.42(1 \mathrm{H}, \mathrm{d}, J=6.0$ $\mathrm{Hz}), 4.36(1 \mathrm{H}, \mathrm{dd}, J=11.0,3.0 \mathrm{~Hz}), 4.18(1 \mathrm{H}, \mathrm{dd}, J=11.0,6.5$ $\mathrm{Hz}), 3.69(1 \mathrm{H}, \mathrm{m}), 2.38$ (1H, m), 1.98 (1H, m), $1.91(1 \mathrm{H}, \mathrm{m}), 1.55$ $(1 \mathrm{H}, \mathrm{m}), 1.02(3 \mathrm{H}, \mathrm{s}), 1.00(3 \mathrm{H}, \mathrm{s}), 0.83(3 \mathrm{H}, \mathrm{s}) .{ }^{13} \mathrm{C}-\mathrm{NMR}(125$ MHz, methanol- $\left.d_{4}\right): \delta 180.3,168.9,142.7,132.2,132.2,130.2$, 130.2, 122.3, 93.0, 75.0, 74.0, 67.7, 56.0, 55.4, 31.5, 30.0, 17.1, 17.0, 9.9; HRESIMS refer to compounds 3a.

$(2 R, 3 S)-3-(4-B r o m o p h e n y l)-2,3-d i h y d r o x y p r o p y l(1 S, 4 R)-4,7,7-$ trimethyl-3-oxo-2-oxabicyclo[2.2.1] heptane-1-carboxylate (6a). The synthesis procedure of 6 a was same with the M1a. Colorless oil; ${ }^{1} \mathrm{H}-\mathrm{NMR}\left(500 \mathrm{MHz}\right.$, methanol- $\left.d_{4}\right): \delta 7.50(2 \mathrm{H}, \mathrm{d}, J=8.5 \mathrm{~Hz})$, $7.34(2 \mathrm{H}, \mathrm{d}, J=8.5 \mathrm{~Hz}), 4.57(1 \mathrm{H}, \mathrm{d}, J=6.5 \mathrm{~Hz}), 4.37(1 \mathrm{H}, \mathrm{dd}, J=$ 11.5, 4.0 Hz), 4.35 (1H, dd, $J=11.5,6.0 \mathrm{~Hz}), 3.90(1 \mathrm{H}, \mathrm{m}), 2.48$ (1H, m), $2.03(1 \mathrm{H}, \mathrm{m}), 1.97(1 \mathrm{H}, \mathrm{m}), 1.62(1 \mathrm{H}, \mathrm{m}), 1.10(3 \mathrm{H}, \mathrm{s})$, $1.09(3 \mathrm{H}, \mathrm{s}), 0.94(3 \mathrm{H}, \mathrm{s}) .{ }^{1} \mathrm{H}-\mathrm{NMR}\left(500 \mathrm{MHz}, \mathrm{DMSO}-d_{6}\right): \delta 7.51$ $(2 \mathrm{H}, \mathrm{d}, J=8.5 \mathrm{~Hz}), 7.30(2 \mathrm{H}, \mathrm{d}, J=8.5 \mathrm{~Hz}), 4.43(1 \mathrm{H}, \mathrm{d}, J=6.5$ 
$\mathrm{Hz}), 4.28(1 \mathrm{H}, \mathrm{dd}, J=11.5,3.5 \mathrm{~Hz}), 4.23(1 \mathrm{H}, \mathrm{dd}, J=11.5,6.5$ $\mathrm{Hz}), 3.69$ (1H, m), 2.37 (1H, m), 1.97 (1H, m), 1.91 (1H, m), 1.54 (1H, m), 1.00 (6H, overlap), $0.84(3 \mathrm{H}, \mathrm{s}) .{ }^{13} \mathrm{C}-\mathrm{NMR}(125 \mathrm{MHz}$, methanol- $\left.d_{4}\right): \delta 180.3,168.9,142.7,132.2,132.2,130.1,130.1$, 122.3, 93.0, 75.1, 74.0, 67.7, 56.0, 55.4, 31.5, 30.0, 17.1, 17.0, 9.9; HRESIMS refer to compounds 3a.

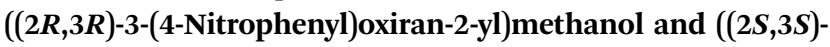
3-(4-nitrophenyl)oxiran-2-yl) methanol. The asymmetric epoxidation was also carried out according to the Sharpless asymmetric Epoxidation. Colorless oil; the specific rotations were

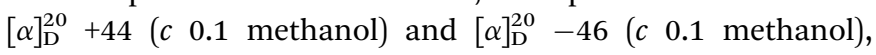
respectively. ${ }^{1} \mathrm{H}-\mathrm{NMR}\left(500 \mathrm{MHz}\right.$, methanol- $\left.d_{4}\right): \delta 8.21(2 \mathrm{H}, \mathrm{d}, J=$ $9.0 \mathrm{~Hz}), 7.53(2 \mathrm{H}, \mathrm{d}, J=9.0 \mathrm{~Hz}), 4.00(1 \mathrm{H}, \mathrm{d}, J=2.0 \mathrm{~Hz}), 3.89$ $(1 \mathrm{H}, \mathrm{dd}, J=13.0,3.0 \mathrm{~Hz}), 3.71(1 \mathrm{H}, \mathrm{dd}, J=13.0,4.5 \mathrm{~Hz}), 3.17$ $(1 \mathrm{H}, \mathrm{m}) ;{ }^{13} \mathrm{C}-\mathrm{NMR}\left(125 \mathrm{MHz}\right.$, methanol- $\left.d_{4}\right): \delta 149.1,146.6,127.7$, 127.7, 124.6, 124.6, 64.5, 62.3, 55.7; HRESIMS $m / z$ 196.0604 [M + $\mathrm{H}]^{+}$(calcd for $\mathrm{C}_{9} \mathrm{H}_{10} \mathrm{O}_{4} \mathrm{~N}$ 196.0608).

$(1 R, 2 S)$-1-(4-Nitrophenyl)propane-1,2,3-triol and $(1 S, 2 R)-1$ (4-nitrophenyl)propane-1,2,3-triol (7 and 8). A solution of $((2 R, 3 R)$-3-(4-nitrophenyl)oxiran-2-yl)methanol (80 $\mathrm{mg}, \quad 0.25$ $\mathrm{mmol}$ ) (which was dissolved by THF) and acetic acid $(0.5 \mathrm{ml})$ in water was stirred at $80{ }^{\circ} \mathrm{C}$ until the solution was dry. Through the analysis of HPLC, the ((2R,3R)-3-(4-nitrophenyl)oxiran-2-yl) methanol was completely hydrolyzed. The residue was separated by chiral column on preparative HPLC ( $n$-hexane/ isopropanol, $80: 20, \mathrm{v} \backslash \mathrm{v})$ to afford $7(50 \mathrm{mg})$ and $8(30 \mathrm{mg})$. The hydrolysis of ((2S,3S)-3-(4-nitrophenyl)oxiran-2-yl)methanol was also produce compounds 7 and $\mathbf{8}$. Compounds 7 and 8 are known compounds and they were identified by comparing their spectral data with those of the known compounds. ${ }^{29}$ Colorless oil; the specific rotations were $[\alpha]_{\mathrm{D}}^{20}-14\left(\begin{array}{ll}c & 0.3\end{array}\right.$ acetone) and $[\alpha]_{\mathrm{D}}^{20}+15$ (c 0.3 acetone), respectively. ${ }^{1} \mathrm{H}-\mathrm{NMR}(500 \mathrm{MHz}$, methanol- $\left.d_{4}\right): \delta 8.23(2 \mathrm{H}, \mathrm{d}, J=8.5 \mathrm{~Hz}), 7.66(2 \mathrm{H}, \mathrm{d}, J=8.5 \mathrm{~Hz})$, $4.74(1 \mathrm{H}, \mathrm{d}, J=7.0 \mathrm{~Hz}), 3.74(1 \mathrm{H}, \mathrm{m}), 3.68(1 \mathrm{H}, \mathrm{dd}, J=11.5,4.5$ $\mathrm{Hz}), 3.65(1 \mathrm{H}, \mathrm{dd}, J=11.5,6.0 \mathrm{~Hz}) .{ }^{1} \mathrm{H}-\mathrm{NMR}(500 \mathrm{MHz}, \mathrm{DMSO}-$ $\left.d_{6}\right): \delta 8.17(2 \mathrm{H}, \mathrm{d}, J=8.5 \mathrm{~Hz}), 7.60(2 \mathrm{H}, \mathrm{d}, J=8.5 \mathrm{~Hz}), 4.72(1 \mathrm{H}, \mathrm{d}$, $J=5.5 \mathrm{~Hz}), 3.52(1 \mathrm{H}, \mathrm{m}), 3.42$ (2H, overlap). ${ }^{1} \mathrm{H}-\mathrm{NMR}(500 \mathrm{MHz}$, acetone- $\left.d_{6}\right): \delta 8.19(2 \mathrm{H}, \mathrm{d}, J=8.5 \mathrm{~Hz}), 7.70(2 \mathrm{H}, \mathrm{d}, J=8.5 \mathrm{~Hz})$, $4.82(1 \mathrm{H}, \mathrm{t}, J=6.0 \mathrm{~Hz}), 3.75(1 \mathrm{H}, \mathrm{m}), 3.67(1 \mathrm{H}, \mathrm{dd}, J=11.0,5.0$ $\mathrm{Hz}), 3.63(1 \mathrm{H}, \mathrm{dd}, J=11.0,5.0 \mathrm{~Hz}) .{ }^{13} \mathrm{C}-\mathrm{NMR}(125 \mathrm{MHz}, \mathrm{DMSO}-$ $\left.d_{6}\right): \delta 151.8,146.4,128.5,128.5,122.6,122.6,75.2,72.9,62.9$; found 258.0619. HRESIMS $\mathrm{m} / z$ 258.0619 $\left[\mathrm{M}+\mathrm{HCOO}^{+}\right.$(calcd for $\left.\mathrm{C}_{10} \mathrm{H}_{12} \mathrm{O}_{7} \mathrm{~N} 258.0620\right)$.

$(2 S, 3 R)-2,3-D i h y d r o x y-3-(4-n i t r o p h e n y l) p r o p y l(1 S, 4 R)-4,7,7-$ trimethyl-3-oxo-2-oxabicyclo[2.2.1] heptane-1-carboxylate (7a). The synthesis procedure of 7a was same with the M1a. Colorless oil; ${ }^{1} \mathrm{H}-\mathrm{NMR}\left(500 \mathrm{MHz}\right.$, methanol- $\left.d_{4}\right): \delta 8.23(2 \mathrm{H}, \mathrm{d}, J=8.5 \mathrm{~Hz})$, $7.66(2 \mathrm{H}, \mathrm{d}, J=8.5 \mathrm{~Hz}), 4.72(1 \mathrm{H}, \mathrm{d}, J=7.0 \mathrm{~Hz}), 4.46(1 \mathrm{H}, \mathrm{dd}, J=$ 11.5, 3.5 Hz), 4.35 (1H, dd, $J=11.5,6.5 \mathrm{~Hz}), 3.91(1 \mathrm{H}, \mathrm{m}), 2.50$ $(1 \mathrm{H}, \mathrm{m}), 2.03(1 \mathrm{H}, \mathrm{m}), 1.98(1 \mathrm{H}, \mathrm{m}), 1.63(1 \mathrm{H}, \mathrm{m}), 1.11(3 \mathrm{H}, \mathrm{s})$, $1.09(3 \mathrm{H}, \mathrm{s}), 0.94(3 \mathrm{H}, \mathrm{s}) .{ }^{1} \mathrm{H}-\mathrm{NMR}\left(500 \mathrm{MHz}, \mathrm{DMSO}-d_{6}\right): \delta 8.20$ $(2 \mathrm{H}, \mathrm{d}, J=9.0 \mathrm{~Hz}), 7.62(2 \mathrm{H}, \mathrm{d}, J=9.0 \mathrm{~Hz}), 4.58(1 \mathrm{H}, \mathrm{dd}, J=6.5$, $3.5 \mathrm{~Hz}), 4.39(1 \mathrm{H}, \mathrm{dd}, J=11.5,3.0 \mathrm{~Hz}), 4.22(1 \mathrm{H}, \mathrm{dd}, J=11.5,6.5$ $\mathrm{Hz}), 3.73$ (1H, m), 2.39 (1H, m), 1.98 (1H, m), $1.91(1 \mathrm{H}, \mathrm{m}), 1.55$ (1H, m), 1.03 (3H, s), $1.00(3 \mathrm{H}, \mathrm{s}), 0.83$ (3H, s). ${ }^{13} \mathrm{C}-\mathrm{NMR}(125$ MHz, methanol- $\left.d_{4}\right): \delta 180.3,168.9,151.4,148.8,129.3,129.3$, 124.1, 124.1, 92.9, 74.8, 74.0, 67.6, 56.0, 55.4, 31.5, 29.9, 17.0,
17.0, 9.9; HRESIMS $m / z 394.1496[\mathrm{M}+\mathrm{H}]^{+}$(calcd for $\mathrm{C}_{19} \mathrm{H}_{24} \mathrm{O}_{8} \mathrm{~N}$ 394.1494).

$(2 R, 3 S)-2,3-D i h y d r o x y-3-(4-n i t r o p h e n y l) p r o p y l(1 S, 4 R)-4,7,7-$ trimethyl-3-oxo-2-oxabicyclo[2.2.1] heptane-1-carboxylate (8a). The synthesis procedure of $8 \mathbf{a}$ was same with the M1a. Colorless oil; ${ }^{1} \mathrm{H}-\mathrm{NMR}$ (500 MHz, methanol- $\left.d_{4}\right): \delta 8.23(2 \mathrm{H}, \mathrm{d}, J=8.5 \mathrm{~Hz})$, $7.66(2 \mathrm{H}, \mathrm{d}, J=8.5 \mathrm{~Hz}), 4.71(1 \mathrm{H}, \mathrm{d}, J=7.5 \mathrm{~Hz}), 4.42(1 \mathrm{H}, \mathrm{dd}, J=$ 11.5, 4.0 Hz), 4.39 (1H, dd, $J=11.5,5.5 \mathrm{~Hz}), 3.92(1 \mathrm{H}, \mathrm{m}), 2.50$ $(1 \mathrm{H}, \mathrm{m}), 2.03(1 \mathrm{H}, \mathrm{m}), 1.97(1 \mathrm{H}, \mathrm{m}), 1.62(1 \mathrm{H}, \mathrm{m}), 1.10(3 \mathrm{H}, \mathrm{s})$, 1.09 (3H, s), 0.95 (3H, s). ${ }^{1} \mathrm{H}-\mathrm{NMR}\left(500 \mathrm{MHz}, \mathrm{DMSO}-d_{6}\right): \delta 8.20$ $(2 \mathrm{H}, \mathrm{d}, J=9.0 \mathrm{~Hz}), 7.63(2 \mathrm{H}, \mathrm{d}, J=9.0 \mathrm{~Hz}), 4.59(1 \mathrm{H}, \mathrm{d}, J=7.0$ $\mathrm{Hz}), 4.31(1 \mathrm{H}, \mathrm{dd}, J=11.5,3.5 \mathrm{~Hz}), 4.26(1 \mathrm{H}, \mathrm{dd}, J=11.5,6.5$ $\mathrm{Hz}), 3.74(1 \mathrm{H}, \mathrm{m}), 2.38(1 \mathrm{H}, \mathrm{m}), 1.97(1 \mathrm{H}, \mathrm{m}), 1.91(1 \mathrm{H}, \mathrm{m}), 1.54$ (1H, m), 1.01 (3H, s), 1.00 (3H, s), 0.84 (3H, s). ${ }^{13}$ C-NMR (125 MHz, methanol- $\left.d_{4}\right): \delta 180.3,168.9,151.4,148.8,129.2,129.2$, 124.2, 124.2, 92.9, 74.9, 74.1, 67.6, 56.0, 55.4, 31.5, 30.0, 17.0, 17.0, 9.9; HRESIMS refer to compound $7 \mathbf{a}$.

$(1 R, 2 R)$-1-(4-Hydroxy-3-methoxyphenyl)propane-1,2,3-triol and $(1 S, 2 S)$-1-(4-hydroxy-3-methoxyphenyl)propane-1,2,3-triol (9 and 10). Colorless oil; the specific rotations were $[\alpha]_{\mathrm{D}}^{20}-19$ (c 0.1 methanol) and $[\alpha]_{\mathrm{D}}^{20}+23$ (c 0.1 methanol), respectively. ${ }^{1} \mathrm{H}-$ NMR (500 MHz, methanol- $\left.d_{4}\right): \delta 6.99(1 \mathrm{H}, \mathrm{d}, J=1.5 \mathrm{~Hz}), 6.80$ $(1 \mathrm{H}, \mathrm{dd}, J=8.5,1.5 \mathrm{~Hz}), 6.76(1 \mathrm{H}, \mathrm{d}, J=8.5 \mathrm{~Hz}), 4.52(1 \mathrm{H}, \mathrm{d}, J=$ $6.5 \mathrm{~Hz}), 3.86(3 \mathrm{H}, \mathrm{s}), 3.66(1 \mathrm{H}, \mathrm{m}), 3.47(1 \mathrm{H}, \mathrm{dd}, J=11.0,4.0 \mathrm{~Hz})$, 3.34 (1H, dd, $J=11.0,6.5 \mathrm{~Hz}$ ). ${ }^{1} \mathrm{H}-\mathrm{NMR}$ (500 MHz, DMSO- $d_{6}$ ): $\delta 6.89$ (1H, brs), 6.69 (2H, overlap), $4.38(1 \mathrm{H}, \mathrm{d}, J=5.0 \mathrm{~Hz}), 3.74$ $(3 \mathrm{H}, \mathrm{s}), 3.44(1 \mathrm{H}, \mathrm{m}), 3.31(1 \mathrm{H}, \mathrm{dd}, J=11.0,4.0 \mathrm{~Hz}), 3.14(1 \mathrm{H}, \mathrm{dd}$, $J=11.0,6.0 \mathrm{~Hz}) .{ }^{13} \mathrm{C}-\mathrm{NMR}\left(125 \mathrm{MHz}, \mathrm{DMSO}-d_{6}\right): \delta 147.0,145.3$, 134.3, 119.1, 114.7, 111.0, 75.9, 72.9, 62.6, 55.6; HRESIMS $\mathrm{m} / \mathrm{z}$ $237.0733[\mathrm{M}+\mathrm{Na}]^{+}$(calcd for $\mathrm{C}_{10} \mathrm{H}_{14} \mathrm{O}_{5} \mathrm{Na}$ 237.0719). Compounds $\mathbf{9}$ and $\mathbf{1 0}$ are known compounds and they were identified by comparing their spectral data with those of the known compounds. ${ }^{7}$

4-((1S,2S)-1,2-Dihydroxy-3-(((1S,4R)-4,7,7-trimethyl-3-oxo-2oxabicyclo[2.2.1] heptane-1-carbonyl)oxy)propyl)-2-methoxyphenyl(1S,4R)-4,7,7-trimethyl-3-oxo-2-oxabicyclo[2.2.1]

heptane-1-carboxylate (10a). Compound 10 (3 mg, $0.014 \mathrm{mmol})$ solution in dry $\mathrm{CH}_{2} \mathrm{Cl}_{2}$ or THF was added to a solution of camphanic acid chloride (6 $\mathrm{mg}, 0.028 \mathrm{mmol})$, EDCI $(5.3 \mathrm{mg}$, $0.028 \mathrm{mmol})$ and DMAP $(1.7 \mathrm{mg}, 0.014 \mathrm{mmol})$ at $0{ }^{\circ} \mathrm{C}$ with the protection of argon gas. The reaction was stirred at $0{ }^{\circ} \mathrm{C}$ for $1 \mathrm{~h}$ and was detected by TLC. Then, the reaction was quenched by dropwise addition of $\mathrm{H}_{2} \mathrm{O}$. The suspension was extracted with $\mathrm{CH}_{2} \mathrm{Cl}_{2}$. Removal of the solvent under reduced pressure and purification of the residue by preparative HPLC, eluting with $55 \%$ methanol $/ \mathrm{H}_{2} \mathrm{O}$, gave the $10 a 1.0 \mathrm{mg}$. Colorless oil; ${ }^{1} \mathrm{H}-\mathrm{NMR}$ $\left(500 \mathrm{MHz}\right.$, methanol- $\left.d_{4}\right): \delta 7.23(1 \mathrm{H}, \mathrm{d}, J=1.5 \mathrm{~Hz}), 7.08(1 \mathrm{H}, \mathrm{d}, J$ $=8.0 \mathrm{~Hz}), 7.02(1 \mathrm{H}, \mathrm{dd}, J=8.0,1.5 \mathrm{~Hz}), 4.71(1 \mathrm{H}, \mathrm{d}, J=5.0 \mathrm{~Hz})$, $4.34(1 \mathrm{H}, \mathrm{dd}, J=11.5,4.0 \mathrm{~Hz}), 4.11(1 \mathrm{H}, \mathrm{dd}, J=11.5,6.5 \mathrm{~Hz})$, $3.96(1 \mathrm{H}, \mathrm{m}), 3.86(3 \mathrm{H}, \mathrm{s}), 2.65(1 \mathrm{H}, \mathrm{m}), 2.49(1 \mathrm{H}, \mathrm{m}), 2.16(2 \mathrm{H}$, overlap), 2.02 (2H, overlap), $1.70(1 \mathrm{H}, \mathrm{m}), 1.63(1 \mathrm{H}, \mathrm{m}), 1.21(3 \mathrm{H}$, s), $1.14(3 \mathrm{H}, \mathrm{s}), 1.11(3 \mathrm{H}, \mathrm{s}), 1.09(3 \mathrm{H}, \mathrm{s}), 1.09(3 \mathrm{H}, \mathrm{s}), 0.94(3 \mathrm{H}, \mathrm{s})$; HRESIMS $m / z 575.2487[\mathrm{M}+\mathrm{H}]^{+}$(calcd for $\mathrm{C}_{30} \mathrm{H}_{39} \mathrm{O}_{11} 575.2490$ ).

Compound 11. White powder; ${ }^{1} \mathrm{H}-\mathrm{NMR}(500 \mathrm{MHz}, \mathrm{DMSO}-$ $\left.d_{6}\right): \delta 6.88$ (2H, overlap), 6.64 (2H, brs), 5.41 (2H, overlap), 4.56 $(1 \mathrm{H}, \mathrm{d}, J=6.0 \mathrm{~Hz}), 4.40(1 \mathrm{H}, \mathrm{d}, J=7.5 \mathrm{~Hz}), 3.77(3 \mathrm{H}, \mathrm{s}), 3.73(6 \mathrm{H}$, s), $3.68(2 \mathrm{H}, \mathrm{m}), 3.64(1 \mathrm{H}, \mathrm{m}), 3.59(1 \mathrm{H}, \mathrm{m}), 3.46(1 \mathrm{H}, \mathrm{m}), 3.38$ 
(2H, overlap), 3.17 (2H, overlap), 3.06 (3H, overlap). ${ }^{13} \mathrm{C}-\mathrm{NMR}$ $\left(125 \mathrm{MHz}\right.$, methanol- $\left.d_{4}\right): \delta$ 147.9, 147.9, 146.6, 142.9, 135.3, 133.5, 131.3, 128.7, 115.7, 112.2, 104.2, 103.7, 103.7, 87.3, 82.0, 76.9, 76.5, 75.2, 74.2, 70.0, 62.7, 61.9, 61.0, 56.0, 56.0, 55.7, 53.1; HRESIMS $m / z$ 607.2026 $[\mathrm{M}+\mathrm{Na}]^{+}$(calcd for $\mathrm{C}_{27} \mathrm{H}_{36} \mathrm{O}_{14} \mathrm{Na}$ 607.2000).

\section{Conclusions}

For natural AGs, there was previously no efficient method to determine their absolute configurations. Our present study presents a convenient and quick method for determining the relative and absolute configurations that only depends on the chemical shift difference $\left(\Delta \delta_{\mathrm{H} 3 \mathrm{a}-\mathrm{H} 3 \mathrm{~b}}\right)$ of the diastereotopic methylene protons (H-3) in ${ }^{1} \mathrm{H}$ NMR spectroscopy. In particular, the method is a good way to determine the limited amounts of natural AGs; such limited amounts prevent the use of other strategies. Remarkably, the empirical rule is invalid in $\mathrm{CDCl}_{3}$. In addition, the introduction of an economic camphanoyl group with larger steric hindrance in this method may provide a reference for solving the stereochemistry problems of other flexible conformer compounds.

\section{Conflicts of interest}

There are no conflicts to declare.

\section{Acknowledgements}

This project was funded by the National Natural Science Foundation of China (No. 81973194) and the Drug Innovation Major Project (No. 2018ZX09711001-008). We appreciate Ms. Y. H. Wang (Institute of Materia Medica, Chinese Academy of Medical Sciences and Peking Union Medical College) for testing the NMR spectra.

\section{References}

1 X. F. Hou, S. Yao, A. Mándi, T. Kurtán, C. P. Tang, C. Q. Ke, X. Q. Li and Y. Ye, Bicunningines A and B, two new dimeric diterpenes from Cunninghamia lanceolate, Org. Lett., 2012, 14, 460 .

2 N. Matsumori, D. Kaneno, M. Murata, H. Nakamura and K. Tachibana, Stereochemical determination of acyclic structures based on carbon-proton spin-coupling constants. a method of configuration analysis for natural products, J. Org. Chem., 1999, 64, 866.

3 S. Higashibayashi and Y. Kishi, Assignment of the relative and absolute configurations of acyclic secondary 1,2-diols, Tetrahedron, 2004, 60, 11977.

4 K. Xu, P. F. Yang, Y. N. Yang, Z. M. Feng, J. S. Jiang and P. C. Zhang, Direct assignment of the threo and erythro configurations in polyacetylene glycosides by ${ }^{1} \mathrm{H}$ NMR spectroscopy, Org. Lett., 2017, 19, 686.

5 S. Y. Shao, F. Zhang, Y. N. Yang, Z. M. Feng, J. S. Jiang and P. C. Zhang, An approach for determining the absolute configuration of $\mathrm{C}-2$ in 2-oxygenated phenylethanoid glycosides by ${ }^{1} \mathrm{H}$ NMR spectroscopy, Org. Lett., 2016, 18, 4084.

6 T. Deyama, T. Ikawa, S. Kitagawa and S. Nishibe, The constituents of Eucommia ulmoides OLIV. V. isolation of dihydroxydehydrodiconiferyl alcohol isomers and phenolic compounds, Chem. Pharm. Bull., 1987, 35, 1785.

7 T. Ishikawa, E. Fujimatu and J. Kitajima, Water-soluble constituents of anise: new glucosides of anethole glycol and its related compounds, Chem. Pharm. Bull., 2002, 50, 1460.

8 H. Matsuura, H. Miyazaki, C. Asakawa, M. Amano, T. Yoshihara and J. Mizutani, Isolation of $\alpha$-glusosidase inhibitors from hyssop (Hyssopus officinalis), Phytochemistry, 2004, 65, 91.

9 S. Lin, S. J. Wang, M. T. Liu, M. L. Gan, S. Li, Y. C. Yang, Y. H. Wang, W. Y. He and J. G. Shi, Glycosides from the stem bark of Fraxinus sieboldiana, J. Nat. Prod., 2007, 70, 817. 10 M. L. Gan, Y. L. Zhang, S. Lin, M. T. Liu, W. X. Song, J. C. Zi, Y. C. Yang, X. N. Fan, J. G. Shi, J. F. Hu, J. D. Sun and N. H. Chen, Glycosides from the root of Iodes cirrhosa, J. Nat. Prod., 2008, 71, 647.

11 X. Y. Chai, H. Y. Ren, Z. R. Xu, C. C. Bai, F. R. Zhou, S. K. Ling, X. P. Pu, F. F. Li and P. F. Tu, Investigation of two Flacourtiaceae plants: Bennettiodendron leprosipes and Flacourtia ramontchi, Planta Med., 2009, 75, 1246.

12 L. Wang, F. Li, C. Y. Yang, A. A. Khan, X. Liu and M. K. Wang, Neolignans, lignans and glycoside from the fruits of Melia toosendan, Fitoterapia, 2014, 99, 92.

13 Y. L. Li, Y. X. Gao, H. Z. Jin, L. Shan, W. L. Chang, X. W. Yang, H. W. Zeng, N. Wang, A. Steinmetz and W. D. Zhang, Chemical constituents of Abies fabri, Phytochemistry, 2015, 117, 135.

14 X. X. Huang, M. Bai, L. Zhou, L. L. Lou, Q. B. Liu, Y. Zhang, L. Z. Li and S. J. Song, Food byproducts as a new and cheap source of bioactive compounds: lignans with antioxidant and anti-inflammatory properties from Crataegus pinnatifida seeds, J. Agric. Food Chem., 2015, 63, 7252.

15 F. H. Li, J. Zhang, M. B. Lin, X. M. Su, C. K. Li, H. Q. Wang, B. M. Li, R. Y. Chen and J. Kang, Anti-inflammatory terpenes from Schefflera rubriflora C. J. Tseng \& G. Hoo with their TNF$\alpha$ and IL-6 inhibitory activities, Phytochemistry, 2019, 163, 23.

16 P. Chakraborty, S. Jana, S. Saha and S. C. Roy, Titanocene (III) chloride mediated formal synthesis of magnofargesin and 7'-epimagnofargesin, Tetrahedron Lett., 2012, 53, 6584.

17 K. Hernández, T. Parella, J. Joglar, J. Bujons, M. Pohl and P. Clapés, Role of the bridge in photoinduced electron transfer in porphyrin-fullerene dyads, Chem.-Eur. J., 2015, 21, 1.

18 A. R. Patel, X. G. Hu, A. Lawer, M. I. Ahmed, C. Au, R. Jwad, J. Trinh, C. Gonzalez, E. Hannah, M. M. Bhadbhade and L. Hunter, Scalable, stereoselective syntheses of $\alpha, \beta$ difluoro- $\gamma$-amino acids, Tetrahedron, 2016, 72, 3305.

19 D. M. M. Barrett, A. R. Neal, C. Hand, J. R. D. Montgomery, I. Panovic, O. S. Ojo, C. S. Lancefield, D. B. Cordes, A. M. Z. Slawin, T. Lebl and N. J. Westwood, The synthesis and analysis of lignin-bound Hibbert ketone structures in technical lignins, Org. Biomol. Chem., 2016, 14, 10023. 
20 D. M. M. Barrett, J. R. D. Montgomery, C. S. Lancefield, D. B. Cordes, A. M. Z. Slawin, T. Lebl, R. Carr and N. J. Westwood, Use of bisulfite processing to generate high- $\beta$-O-4 content water-soluble lignosulfonates, ACS Sustainable Chem. Eng., 2017, 5, 1831.

21 L. Monsigny, E. Feghali, J. C. Berthet and T. Cantat, Efficient reductive depolymerization of hardwood and softwood lignins with Brookhart's iridium (iii) catalyst and hydrosilanes, Green Chem., 2018, 20, 1981.

22 E. Lallana, F. Freire, J. M. Seco, E. Quiñoá and R. Riguera, The ${ }^{1} \mathrm{H}$ NMR method for the determination of the absolute configuration of 1,2,3-prim,sec,sec-triols, Org. Lett., 2006, 8, 4449.

23 F. Freire, E. Lallana, E. Quiñoá and R. Riguera, The Stereochemistry of 1,2,3-triols revealed by ${ }^{1} \mathrm{H}$ NMR spectroscopy: principles and applications, Chem.-Eur. J., 2009, 15, 11963.

24 Y. N. Yang, B. Han, P. F. Yang, Z. M. Feng, J. S. Jiang and P. C. Zhang, A concise approach for determining the relative configuration of $\mathrm{H}-7$ and $\mathrm{H}-8$ in 8,4'-oxyneolignans by ${ }^{1}$ H NMR spectroscopy, Org. Chem. Front., 2019, 6, 886.
25 Y. Gao, R. M. Hanson, J. M. Klunder, S. Y. Ko, H. Masamune and K. B. Sharpless, Catalytic asymmetric epoxidation and kinetic resolution: modified procedures including in situ derivatization, J. Am. Chem. Soc., 1987, 109, 5765.

26 C. S. Philbin and S. J. Schwartz, Resolution of diastereomeric flavonoid $(1 S)-(-)$-camphanic acid esters via reversed-phase HPLC, Phytochemistry, 2007, 68, 1206.

27 C. Meier, W. H. G. Laux and J. W. Bats, Asymmetric synthesis of chiral, nonracemic dialkyl $\alpha$-hydroxyarylmethyl and $\alpha$-, $\beta$ and $\gamma$-hydroxyalkylphosphonates from keto phosphonates, Liebigs Ann. Chem., 1995, 1995, 1963.

28 L. Pisano, L. Degennaro, M. Carraro, U. Azzena, F. Fanelli, P. Mastrorilli and R. Luisi, Computational NMR as useful tool for predicting structure and stereochemistry of fourmembered sulfur heterocycles, Eur. J. Org. Chem., 2016, 2016, 3252.

29 X. Hao, T. Nguyen, D. B. Kearns, C. C. Arpin, R. Fall and T. Sammakia, New inhibitors of colony spreading in Bacillus subtilis and Bacillus anthracis, Bioorg. Med. Chem. Lett., 2011, 21, 5583. 